\title{
Scalable Crystalline Porous Membrane: Current state and Perspectives
}

Received 00th January 20xx, Accepted 00th January 20xx DOI: $10.1039 / \times 0 \times x 00000 x$

\author{
Zixi Kanga, d, Hailing Guo ${ }^{b}$, Lili Fan ${ }^{a}$, Ge Yang ${ }^{b}$, Yang Feng ${ }^{a}$, Daofeng Sun*, a, Svetlana Mintova*,b,
}

The crystalline porous materials (CPMs) with uniform and regular pore systems show great potential for separation applications using the membrane technology. Along with the research on synthesis of precise-engineered porous structures, a significant attention on the practical application of these materials for preparation of crystalline porous membranes (CPMBs) is paid. In this review, the progress made in the preparation of thin, large area and defect-free CPMBs using classical and novel porous materials and processing is presented. The current state-of-the-art of scalable CPMBs with different nodes (inorganic, organic and hybrid) and various linking bonds (covalent, coordination, and hydrogen bond) is revealed. The advances made in the scalable production of high-performance crystalline porous membranes are categorized according to the strategies adapted from polymer membranes (interfacial polymerization, solution-casting, melt extruding and polymerization of CPMs) and tailored based on CPMs properties (seeding-secondary growth, conversion of precursors, electrodeposition and chemical vapor deposition). Finally, the performance and challenges in the preparation of scalable CPMBs with emphasis on their sustainability are presented.

\section{Introduction}

Membrane-based separation is recognized as a powerful technology that guarantees high selectivity, low energy consumption and small footprint for separation application, and fulfill the need for a new sustainable industrial process. ${ }^{1,2}$ The research focused on materials development will enable synthetic membranes to be further extended to the development of large-scale, energy-efficient molecular separation technologies. ${ }^{3-7}$ On one hand, the membrane's barrier layer should be well defined, which composes of transport channels with identical pore size and properties that ensure selective permeation. On the other hand, the materials should offer as little resistance as possible by increasing the porosity and declining thickness of barrier layer to achieve high flux. The crystalline porous materials (CPMs) with ordered pore structures with high selectivity and large pore volumes for high flux are highly attractive for membrane separation, ${ }^{8}$ and have been widely studied in the last two decades including (i) the classical zeolites, ${ }^{9}$ (ii) metal-organic frameworks (MOFs), ${ }^{10-12}$ (iii) covalent organic frameworks (COFs), ${ }^{13}, 14$ (iv) porous organic cages $^{15}$ (POCs) and hydrogen-bonded organic frameworks

\footnotetext{
a. School of Materials Science and Engineering, China University of Petroleum (East China), 266580 Qingdao, China. dfsun@upc.edu.cn

b. State Key Laboratory of Heavy Oil Processing, Key Laboratory of Catalysis, China University of Petroleum (East China), 266555 Qingdao, China.

c. Laboratoire Catalyse et Spectrochimie (LCS), Normandie University, ENSICAEN,

CNRS, 6 boulevard du Marechal Juin, 14050 Caen, France. mintova@ensicaen.frb

d. State Key Laboratory of Structural Chemistry, Fujian Institute of Research on the

Structure of Matter, Chinese Academy of Sciences, Fuzhou, Fujian, 350002, PR China.

Electronic Supplementary Information (ESI) available: [details of any supplementary information available should be included here]. See DOI: 10.1039/x0xx00000x
}

(HOFs). ${ }^{16}$ Beyond the uniform pore system, the CPMs also possess other features like tailorable window size and cages, which are the base for constructing of crystalline porous membranes (CPMBs). Considerable numbers of research papers on the CPMBs have been published in recent years. As shown in Fig. 1, the number of publications dedicated on CPMBs has increased significantly during the last two decades; particularly the papers on the scalable preparation of CPMBs in the last ten years increased drastically. The researchers pay more attentions to the practical application of the CPMBs bringing new vigor to the separation industry.
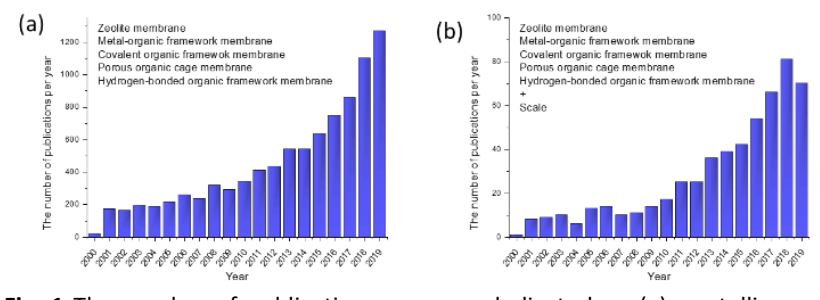

Fig. 1 The number of publications per year dedicated on (a) crystalline porous membranes (CPMBs) and (b) scalable CPMBs. Data obtained from Web of Science until August 13th, 2020.

The CPMBs has been the main focus of several excellent reviews describing the following specific aspects: (1) preparation of different CPMBs using zeolite, MOF, ZIF, COF, CPM based mixed matrix membranes; 9 , 11, 17-21 (2) description of different separation applications including gas separation, reversed osmosis, nanofiltration; ${ }^{22-32}$ and (3) structural design, stability and preparation methods toward CPMBs with 2D morphology. ${ }^{33-38}$ However, as emphasized by Baker "a film is not a membrane", for the practical separation applications. 
Table 1 CPMs applied for preparation of CPMBs: features of composites and structures

\begin{tabular}{|c|c|c|c|c|c|}
\hline CPMs & Zeolites & MOFs & $\begin{array}{c}\text { COFs } \\
\end{array}$ & POCs & 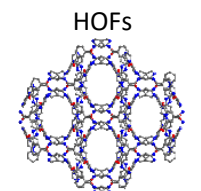 \\
\hline Nodes & Inorganic & $\begin{array}{c}\text { Inorganic- } \\
\text { organic hybrid }\end{array}$ & Organic & Organic & Organic \\
\hline Bonds & Covalent bonds & Coordination bonds & Covalent bonds & $\begin{array}{l}\text { Covalent bonds and } \\
\text { Intermolecular force }\end{array}$ & Hydrogen bonds \\
\hline Bond reversibility & Low & Medium & Low to medium & High & High \\
\hline Structural dimension & $2 D$ and $3 D$ & $2 D$ and $3 D$ & 2D and 3D & $\mathrm{OD}$ & 2D and 3D \\
\hline Stability & High & Medium & Medium to high & Low to medium & Low to medium \\
\hline Structural flexibility & Low & Medium to high & Low to medium & High & High \\
\hline
\end{tabular}

The promising materials must be processed into thin, typically supported membranes, and further produced as modules with a high surface/volume ratio, and then employed and optimized in a real process. Up to date, only a few CPMs have been used in industry, such as zeolite $\mathrm{NaA}$ (LTA framework type zeolite) for organic solvent dewatering. ${ }^{39}$ In 2012, Gascon systematically reviewed the practical issues related with zeolite membranes and more specifically he discussed the reproducibility and scalable synthesis of zeolite membranes. ${ }^{40}$ With the rapid development of new materials and deeper understanding of growth (deposition) processes, a new stage of the CPMBs is reached that requires to summarize the latest progress, developing trends, emerging challenges and opportunities primarily oriented to the scalable production of CPMBs.

This review will present the current view on the CPMB fabrication with high-performance materials for separation purposes. Both the continuity and ordered porous materials are pre-requested and challenging for constructing defect-free CPMBs. The building blocks for CPM should be applied to construct not only the crystalline structure but also thin film for a reasonable time, thus the traditional crystallization process needs to be adapted based on the respective assembly methods used for different CPMs. The CPMs are assembled by repeating units similar to polymers but in a fully ordered way, thus some preparation methods for the CPMBs can be adapted from the polymers, such as the interfacial polymerization (IP) and solution-processing approach. Furthermore, the financial aspects should be kept in mind in terms of the demand at industrial scale. The energy saving process, low waste emission and commercial supports have to be considered as well.

This review addresses the current state-of-the-art of scalable CPMBs with different nodes (inorganic, organic and hybrid) and various linking bonds (covalent, coordination, and hydrogen bond). We will discuss the different CPMs and their features, the strategies adapted from the preparation of polymer membranes and tailored based on the properties of CPMs, and outline the vision on the future scalable membrane preparation bringing these materials closer to implementation for practical applications. The mixed matrix membranes (MMMs) and thinfilm nanocomposite (TFN) membranes consisting of CPMs but determined by the properties of polymers would not be the scope of this review.

\section{Classification of crystalline porous materials (CPMs) and their advantages for scalable production of membranes (CPMBs)}

The CPMs possess uniform pore structures assembled of different nodes that bring advantages for their scalable preparation (Table 1).

\subsection{Zeolites}

Zeolites are crystalline microporous aluminosilicate frameworks built of corner-sharing $\mathrm{TO}_{4}$ tetrahedra $(\mathrm{T}=\mathrm{Si}, \mathrm{Al}) .{ }^{41}$ The size of pores and cages can be varied by synthesizing zeolites with different framework structures, various extra-framework cations, doping with heteroatoms and introducing exchangeable guest molecules. ${ }^{42}$ The covalent bonds guarantee stable and rigid zeolite structures, although a local flexibility in several zeolites with low Si/Al ratio was found. Because of the tunable pore size and excellent stability, zeolite polycrystalline membranes have shown great performance in the field of solvent dehydration, gas separation and desalination. Due to the high bonding energy of covalent bonds, the zeolite materials are generally synthesized under hydrothermal-solvothermal conditions. Continuous polycrystalline zeolite membranes were mostly prepared by seeding approach (Table 2). ${ }^{43}$ The sol-gel conversion under steam conditions is also applied toward largescale growth of zeolite membranes. ${ }^{44}$ The main issues of scalable zeolite membranes are the formation of inter-crystal defects, removal of structural directing agents (SDAs) and high cost of ceramics supports (substrates); ${ }^{45}$ further discussion will be provided in section 4.1.

\subsection{Metal-organic frameworks (MOFs)}

MOFs constructed from metal cations or clusters and organic ligands, are another class of crystalline materials with reticular topology. MOFs materials are constructed with various topologies, functional groups, modifiable adaptors that result in highly tunable pore sizes, pore shape, and polarity. ${ }^{12,}{ }^{46}$ Because the nodes are linked by coordination bonds, MOF structures 
Table 2 Preparation conditions and separation performance of zeolite membranes discussed in this review.

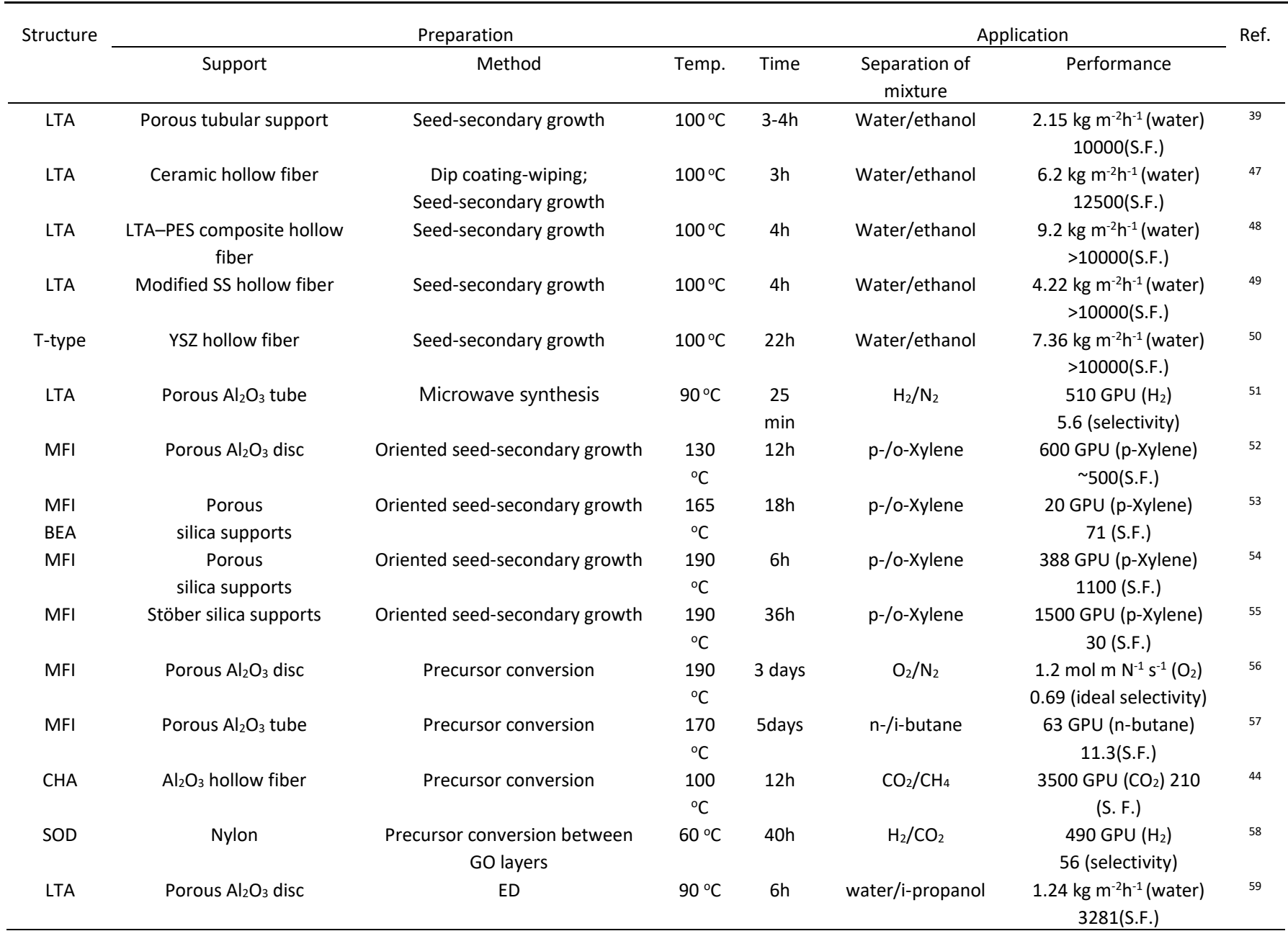

show enhanced flexibility and lower hydrothermal stability compared with zeolites. ${ }^{60}$ The so-called breathing effect is observed in some MOFs such as the MIL series, ${ }^{61}$ which exhibits dynamic aperture as the loading of guest molecules leading to the stimulus-responsive separation performance. ${ }^{62}$ The rising numbers of MOF structures led to further use for separation membranes based on their molecular sieving and chemical affinity. Diversified strategies including interfacial polymerization (IP), chemical vapor deposition (CVD), electrodeposition (ED) and precursor conversion can be applied to scale the preparation of MOF membranes (Table 3). Similar to the zeolite membrane, the defects formed in the MOF membranes are the main concerns for their scalable preparation. ${ }^{20}$ Recently several MOFs were prepared by melting decomposition and transferred into continuous MOF glass membranes by melt extruding. ${ }^{63}$ This led to the preparation of membranes with less defects. Because of the metal centers in structures, the metal based supports are considered to be used as both membrane substrates and metal sources for the MOFs crystallization. ${ }^{64}$ On the other hand, the organic linkers bridge the MOFs with the polymers, forming PolyMOFs. ${ }^{65}$ The stability of the membranes is another problem that hinders the practical application of MOF membrane. Thus more attention is paid on the robustness of MOF frameworks and their stabilization by protective coating layers and post-synthesis treatments. ${ }^{66,67}$

\subsection{Covalent organic frameworks (COFs) and porous aromatic frameworks (PAFs)}

The covalent organic frameworks (COFs) are prepared by topology-diagram-directed polymer growth in conjunction with geometry matching between monomers. ${ }^{13}$ COFs are consisting of full organic building blocks and strong covalent bonds, and they have great variety of structures in comparison to zeolites, and also they exhibit higher stability than the MOFs. The structural design and separation application of COFs have been comprehensively discussed in two recent reviews. ${ }^{13,} 14$ Unlike the linear polymers, the most synthesized COFs are difficult to be dissolved into solvents like cross-linked polymers and conjugated microporous polymers (CMPs). ${ }^{68}$ Therefore the COFs cannot be fabricated into membranes by solutionprocessing technology. However, the different ligands can be dissolved in diverse solvents and form the COFs membranes by 
Table 3 Preparation conditions and separation performance of MOF membranes discussed in this review.

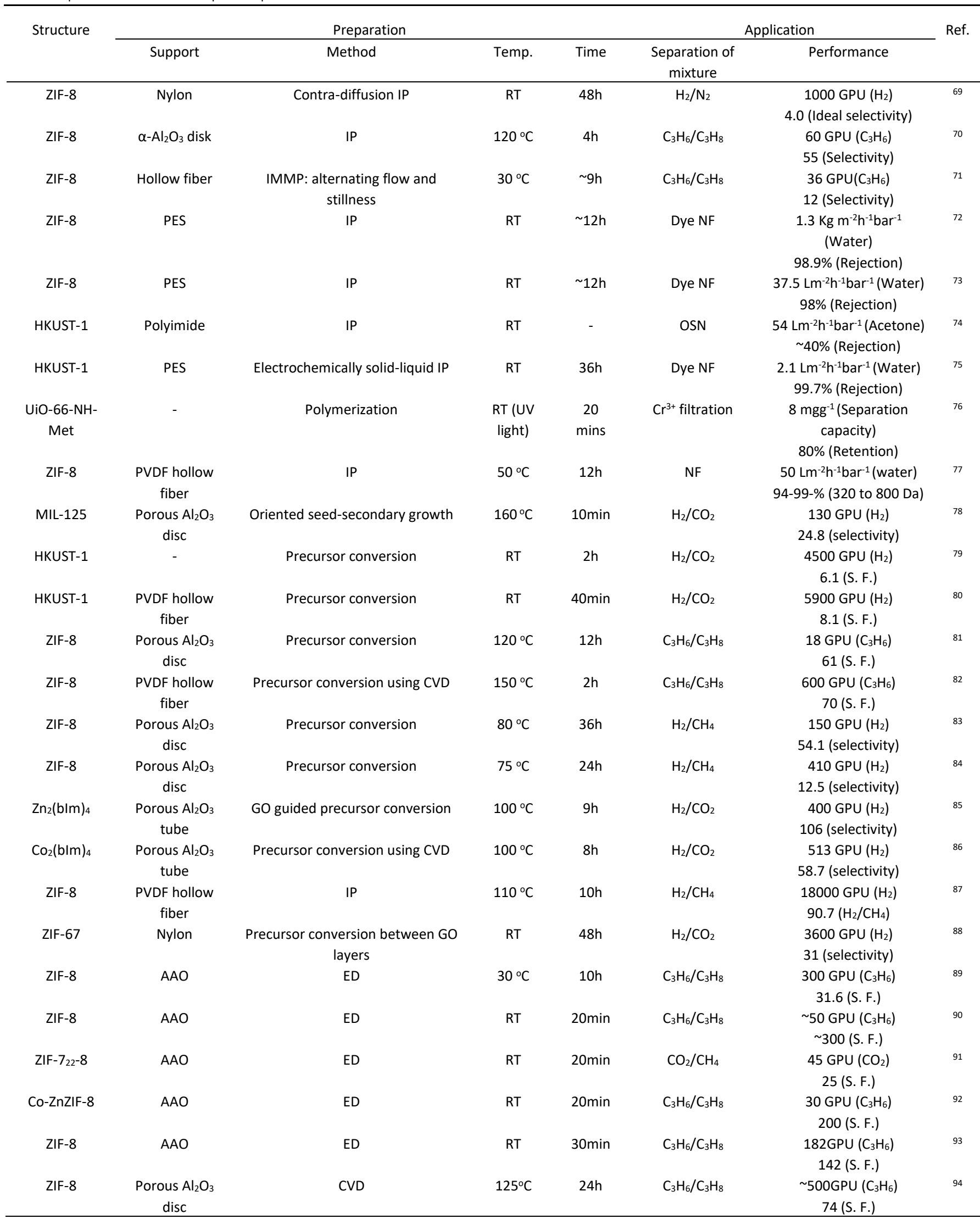


Table 4 Preparation conditions and separation performance of COF membranes discussed in this review.

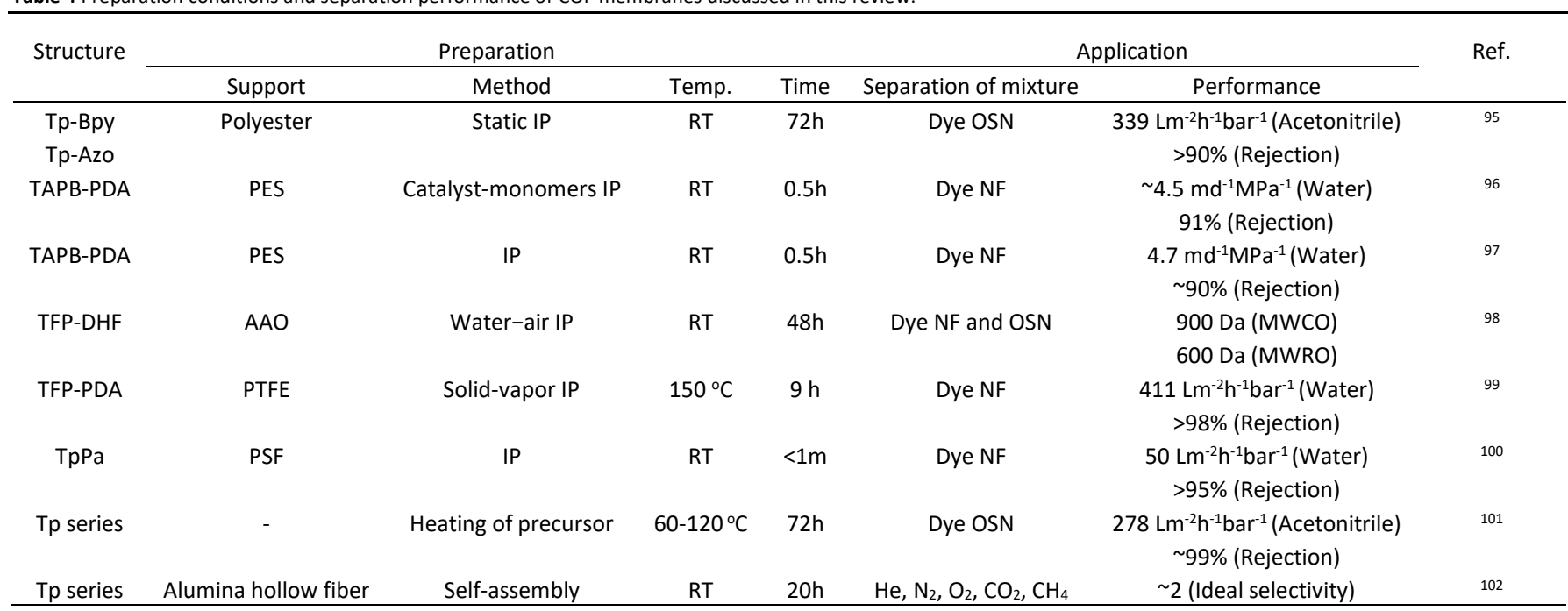

Table 5 Preparation conditions and separation performance of other CPMBs discussed in this review.

\begin{tabular}{|c|c|c|c|c|c|c|c|}
\hline \multirow[t]{2}{*}{ Structure } & \multicolumn{4}{|c|}{ Preparation } & \multicolumn{2}{|c|}{ Application } & \multirow[t]{2}{*}{ Ref } \\
\hline & Support & Method & Temp. & Time & Separation of mixture & Performance & \\
\hline \multirow[t]{2}{*}{$\mathrm{CC} 3, \mathrm{CC} 13$} & AAO & Solution-processing & RT & - & $\mathrm{H}_{2} / \mathrm{N}_{2}$ & $4500 \mathrm{GPU}\left(\mathrm{H}_{2}\right)$ & 103 \\
\hline & & & & & & 30 (Ideal selectivity) & \\
\hline \multirow[t]{2}{*}{ UPC-HOF-6 } & Porous $\mathrm{Al}_{2} \mathrm{O}_{3}$ disc & Solution-processing & $80^{\circ} \mathrm{C}$ & $2 \mathrm{~h}$ & $\mathrm{H}_{2} / \mathrm{N}_{2}$ & $885.1 \mathrm{GPU}\left(\mathrm{H}_{2}\right)$ & 104 \\
\hline & & & & & & 17.2 (selectivity) & \\
\hline \multirow[t]{3}{*}{ ZIF-62 glass } & Porous $\mathrm{Al}_{2} \mathrm{O}_{3}$ disc & Melt-quenching & $440^{\circ} \mathrm{C}$ & $\sim 1 \mathrm{~h}$ & $\mathrm{H}_{2} / \mathrm{CH}_{4} \mathrm{CO}_{2} / \mathrm{N}_{2}$ & $50.7\left(\mathrm{H}_{2} / \mathrm{CH}_{4}\right)$ & 63 \\
\hline & & & & & $\mathrm{CO}_{2} / \mathrm{CH}_{4}$ & $34.5\left(\mathrm{CO}_{2} / \mathrm{N}_{2}\right)$ & \\
\hline & & & & & & $36.6\left(\mathrm{CO}_{2} / \mathrm{CH}_{4}\right)$ & \\
\hline \multirow[t]{2}{*}{ Noria } & PAN & IP & RT & 8 mins & Dye OSN & $18 \mathrm{Lm}^{-2} \mathrm{~h}^{-1} \mathrm{bar}^{-1}$ (Methanol) & 105 \\
\hline & & & & & & 97\% (Rejection) & \\
\hline
\end{tabular}

interfacial polymerization (Table 4). In this process, the epitaxial and continuous assembling of ligands is essential to obtain high quality membranes. The more reversible bonding formation such as boronate-ester-link allowed structural corrections leading to formation of more ordered structure but unstable frameworks. Also learning from the classic polymer science, the nanosized COFs can be polymerized leading to the formation of membranes. The insoluble COFs are suitable for the organic solvent nanofiltration (OSN) approach. ${ }^{98}$

Porous aromatic frameworks (PAFs) have pure organic frameworks linked via irreversible and strong C-C bonds. ${ }^{106}$ Although the PAFs have amorphous frameworks evidenced by $\mathrm{XRD}$, the predesigned building blocks bring higher order and much larger surface area than the polymers. The narrow pore distribution and excellent stability make the PAFs promising materials for membrane separation. PAFs are insoluble in most solvents, similar to MOFs and COFs. Furthermore, the irreversible changes in the bonds of PAFs can lead to the production of powder samples (solids) under kinetic control. Therefore, to process PAFs into MMMs membranes, mostly blending with polymer is used. Taking the advantage of the PAFs with small particle sizes, they may be further interfacial polymerized with common organic monomers to form continuous membranes. The details on PAFs and related membranes can be found in the review article reported by Zhu. ${ }^{107}$

\subsection{Porous organic cages (POCs) and hydrogen-bonded organic frameworks (HOFs)}

As discussed above, zeolites, MOFs and COFs are not soluble in solvents, which creates difficulties in the preparation of membranes. Porous organic cages (POCs) are a relatively new class of CPMs; they are composed of molecules with intrinsic cavities connected by Van der Waals forces, $\pi-\pi$ interaction, and hydrogen bonds. ${ }^{108,} 109$ They have some intriguing differences from extended porous frameworks, such as solution processability, making them attractive for fabrication of membranes (Table 5). Especially the phase inversion (PI) method may be applied for the preparation of POC membranes using poor solvents, because all porous cage molecule can be seen as a polymer chain. The optimization of the preparation process is challenging since different solvents may lead to diverse cage packing. The stability of the POCs membrane for separation purposes is another important issue to be considered (the Achilles' heel). ${ }^{103}$ The intramolecular stability was optimized by using strong imine bond or adding reinforced ligands. The structural transformation of POCs can easily occurred by external stimulus such as removal/introduction of solvents, high temperature, and high pressure leading to high 
reversible interactions between cages. As a result, additional voids can be formed resulting in the loss of selectivity. Postsynthesis treatment can be applied to modify the cages. Moreover, as molecules with intrinsic pores, the POCs can play the role of monomers to construct membrane by IP method. ${ }^{105}$ On the contrary, the hydrogen-bonded organic frameworks (HOFs) are extended frameworks consisting of highly flexible intermolecular hydrogen-bonding interactions. ${ }^{16,} 110$ The flexible HOFs can be easy processed and the self-healing properties can be of great advantage for the preparation of membranes. ${ }^{111}$ The multiple $\mathrm{H}$-bonding interactions between organic ligands, or charge assisted $\mathrm{H}$-bonds between cations and anions, especially the high charged ionic ones, are favorable for the synthesis of stable HOFs.

\section{Strategies adapted from polymer membranes}

\section{toward preparation of CPMBs}

As mentioned by Baker in his famous fundamental book for membrane technology and applications: "First, membrane materials with the appropriate chemical, mechanical, and permeation properties must be selected; this choice is very process specific. However, once the membrane material has been selected, the technology is required to fabricate this material into a robust, thin, defect-free membrane, and then to package the membrane into an efficient, economical, highsurface-area module, which is similar for all membrane processes." $^{1}$

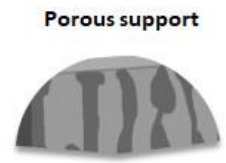

IP for CPMBs:
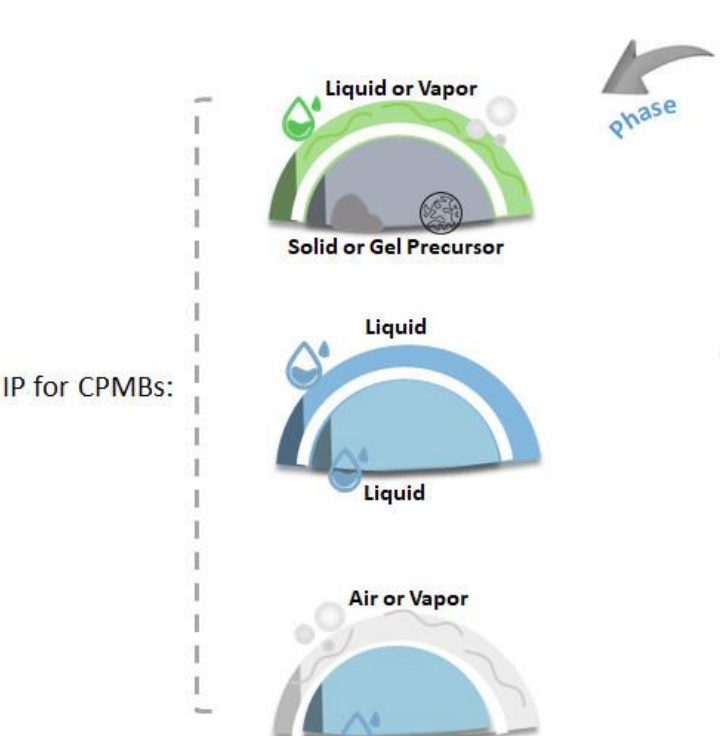

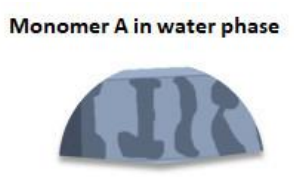

Renovate

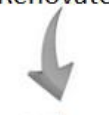

status

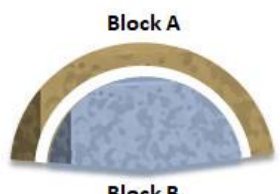

(Static)

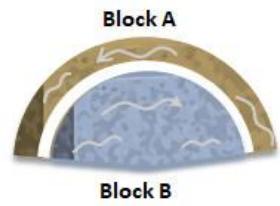

(Fluid)
Monomer B in oil phase

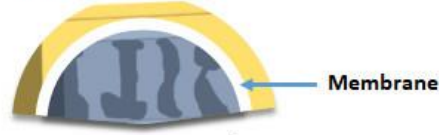

Monomer $\mathrm{A}$ in water phase
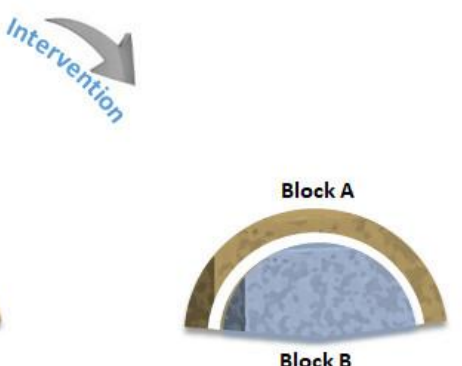

Block B

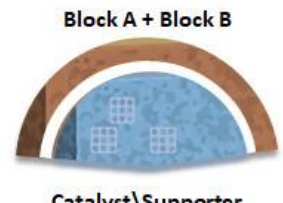

Catalyst \Supporter

Fig. 2 Classical interfacial polymerization (IP) approach applied for fabrication of thin-film composite (TFC) membrane (top) and adapted IP processes for CPMBs (bottom) at different interfaces: (liquid/solid, liquid/liquid and liquid/gas), under various conditions (static, fluid and stepwise). 
reason for the formation of crystalline membrane. Recently developed POC and HOF membranes were prepared by evaporation of solvents, but resulting in the isotropic membranes, which more resemble the solution-casting method of the dense polymer membranes. ${ }^{103,113}$ Thus, the optimization will focus on using of casting solution and specific precipitation media in order to guarantee heterogeneous nucleation and balance with the precipitation rate for phase inversion preparation of continuous CPMBs. The selection of supports with a "fertile" surface may help solving the insufficient nucleation issues and can lead to formation of intergrown homogenous membranes. Another widely used method for preparation of polymer membranes, the interfacial polymerization, has been successfully adapted and applied for fabrication of CPMBs.

\subsection{Interfacial polymerization (IP) approach}

Interfacial polymerization (IP) is the main technology after phase inversion, which is mostly used for production of reversed osmosis (RO) and nanofiltration (NF) membranes. In a typical IP process, different monomers are dissolved in two immiscible solvents, and the dense cross-linked polymer layers are formed at the interface. The "self-inhibited" effect ensures that the monomers only polymerize at the unsealed sites, resulting in thin and defect-free membranes. This strategy is suitable for the preparation of CPMs assemblies with different units that can dissolve in immiscible solvents, such as MOFs and COFs (Fig. 2). ${ }^{114}$

MOFs are constructed with metal based nodes and organic linkers, which are readily dissolved in two solvents with different polarity. The first example for MOF (copper based MOF, HKUST-1) synthesized at an interface was reported by Vos in 2011. ${ }^{115}$ The thin free-standing film and hollow capsules were grown at the interface of an aqueous solution of a copper acetate and a solution of 1,3,5-benzenetricarboxylic acid $\left(\mathrm{H}_{3} \mathrm{BTC}\right)$ in 1-octanol. Although the thin film was not applied for membrane separation, the size-selective permeability of small molecules was reported. In the same year, Wang reported the preparation of MOF membrane by the contra-diffusion synthesis method for gas separation. ${ }^{69}$ The ZIF- 8 membrane was obtained at room temperature for $72 \mathrm{~h}$ on a nylon support that divided the solutions of $\mathrm{Zn}^{2+}$ and 2-methylimidazolate (2-MIM). In both cases revealed above, the porous substrates were in immobile contact with the two solutions. In 2013, Jeong fabricated the ZIF- 8 membrane by immersing the porous $\mathrm{Al}_{2} \mathrm{O}_{3}$ supports in different solutions but in stepwise manner, ${ }^{70}$ which can be regarded as a similar approach to the tradition IP process. In comparison to the previous work, here a shorter time $(4 \mathrm{~h})$ and a higher temperature $\left(120{ }^{\circ} \mathrm{C}\right)$ were used to prepare well-intergrown membrane, which exhibited exceptionally high separation performance toward propylene over propane. For the MOF growth on immobile surface, the high temperature and deprotonating agent such as acetate and formic acid were applied to accelerate the coordination rate and form the continuous membranes. ${ }^{70,74}$

The commercial and low-cost polymers are appropriate supports for scalable IP fabrication of CPMBs. The fluid or traditional two-step IP processes have been investigated. In 2014, Nair reported the ZIF-8 membrane fabricated by interfacial microfluidic membrane processing (IMMP) in a module of hollow fiber. ${ }^{71}$ The flow profile was set as an initial continuous flow followed by static growth phases interrupted only by short reactant replenishment steps, to obtain thin and intergrown membrane for high efficient separation of $\mathrm{C}_{3} \mathrm{H}_{6} / \mathrm{C}_{3} \mathrm{H}_{8}$. The continuous flow offered the adequate nutrition and relatively rapid transport of reactants to the interface, leading to rapid formation of a ZIF-8 layer at room temperature (several hours). The recycling of reactants and the use of hollow fiber substrates are the other advantages for further scale-up of this method. In the work of Vankelecom in 2015, the flexible polyethersulfone polymer (PES) was used as a porous support. ${ }^{72}$ The PES membrane was impregnated with a zinc nitrate aqueous solution overnight and after removal of the excess aqueous solution from the support surface, a solution of hexane with ethanol as co-solvent (2-MIM) was poured on the surface. The solution was drained off after $1 \mathrm{~h}$ reaction, then posttreated at $100{ }^{\circ} \mathrm{C}$ overnight. Because of the poor solubility of $\mathrm{Zn}^{2+}$ and 2-MIM in hexane, the reaction happened toward the aqueous phase, and thus the ZIF-8 grew inside the pores of the support resulting in low water flux for dye NF application. To solve this problem, the same group used octanol instead of hexane. ${ }^{73}$ The membrane formed at the octanol/water interface showed highly improved water flux, while, the membrane grown above the support was brittle and easily delaminated from the surface. As reported by Livingston in 2015, a thin layer of HKUST-1 was synthesized on the polyimide supports by two methods, and evaluated for the organic solvent nanofiltration (OSN) performance. ${ }^{74}$ According to the first method, the support initially was soaked in a water solution of $\mathrm{Cu}^{2+}$, then was immersed in octanol solution of BTC, resulting in the brittle HKUST-1 layer grown on the polymer membrane surface, which did not chemically bond to the polymer support layer beneath. This was caused by the diffusion of $\mathrm{Cu}^{2+}$ into octanol over time, and the HKUST-1 formation reaction occurred just on the surface of the membrane. According to the second methodology, the BTC solution was impregnated in the membrane, the $\mathrm{Cu}^{2+}$ diffused into the membrane, leading to HKUST-1 growth on the surface but also in polymer support membrane. As the HKUST-1 was physically embedded into the polymer layer, thus less cracks and defects were formed.

So far, all the MOF membranes prepared by liquid-liquid IP process just considered structures that can be constructed at room temperature, such as HKUST-1, MOF-5 and ZIF-8. More stable frameworks like UiO and MIL series have not been deposited in membranes by IP process, because the strong bonding interactions cannot be formed at the IP condition that favor the formation of continuous membranes. Therefore novel strategy or optimization of the IP process are needed to facilitate the strong coordination bonds and avoid the formation of discontinuous crystals. The traditional IP process involving treatment of supports in different solvents step by step is preferred, because it is easier to be scaled up.

Another issue that hinders the broader application of IP for MOF membranes is that only a limited number of matching solvents 
can be used. The solvent pair should be immiscible and also can dissolve metal salt and ligand separately. To solve this problem, the binary solvent system (aqueous/organic) can be changed to a single-solvent system, and MOF membranes are fabricated at solid-liquid (metal solid - ligand solution) interface. In the "single metal source" method reported in our previous works, the nickel meshes were used as both the support and $\mathrm{Ni}^{2+}$ source to produce $\mathrm{Ni}$ based chiral MOF membranes for separation of the racemic mixture. ${ }^{64}$ Then in the work reported by Fransaer, an electric field was applied to facilitate the dissolution of metal ions from the copper plane at the anode, then passed through the PES porous support and contacted with the BTC solution to construct the continuous HKUST-1 membrane for dye NF. ${ }^{75}$ Since all the MOFs contain the metal nodes, this innovative strategy is expected to significantly extend the scope of interfacial synthesis of MOFs with varied metal centers on the porous substrate surfaces. Furthermore, the pure metal can be adapted to other metal based precursor, such as oxide and hydroxide, reacting with ligand solution to form large area MOF membranes on different supports, which will be discussed in section 4.2. On the other hand, the solution can be replaced by the vapor phase of ligands as well, and the relevant studies for the CVD preparation of MOF membranes will be surveyed in section 4.4. Another alternative strategy reported by Li recently is to grow the MOF membrane on the liquid-air interface. ${ }^{77}$ Firstly, a precursor aqueous solution containing 2-MIM and $\mathrm{Zn}^{2+}$ was immersed into the polymer hollow fiber. Through water evaporation under mild conditions, the precursor aqueous solution close to air-water interface was concentrated, and then the preferential crystallization at the solution surface in the pores of substrates led to the formation of continuous ZIF-8 membranes, which possessed the rejections of 94.1-99.5\% for small molecules with MW from 320 to $800 \mathrm{Da}$. Moreover, this method can be applied to fabricate the $30 \mathrm{~cm}$ ZIF-8 hollow fiber membrane with the assistant of microfluidic processing, which also exhibit high separation performance.

As discussed above, the COFs can be considered as a crosslinked polymer with the long-range order, thus the main difficulty for using the IP methodology for preparation of COF membranes is to ensure arranged assembly of building blocks. ${ }^{96}$, 97 Based on the published work, we could conclude that mostly the IP process was applied to prepare imine-typed COFs. The assistant of catalyst is a key element to avoid the formation of amorphous polymers during the organic Schiff base reaction. In 2017, Banerjee reported the first COF (Tp series, Tp: 1,3,5triformylphloroglucinol) membranes prepared by IP. ${ }^{95}$ Two monomers Tp and diamine were dissolved in dichloromethane and water, respectively, then polymerized at the liquid-liquid interface and transferred to supports. Before the polymerization, the p-toluene sulfonic acid (PTSA) reacted with the amine to form salt. The $\mathrm{H}$ bonding in the PTSA-amine decreased the diffusion rate of amine organic linkers, and the reaction rate was thermodynamically controlled. However, different from polymer, COFs have the regular framework. The polymeric membrane formed after 24 hours needed more time (another 48 hours) to achieve a certain degree of crystallinity. To reduce the reaction time and membrane thickness, Jiang reported the IP preparation of COF membrane at solid/vapor interface. ${ }^{99}$ Similar to the process of MOF membranes, one monomer was firstly spin-coated onto the substrate, then reacted with the vapor of another monomer at $150{ }^{\circ} \mathrm{C}$ for 9 hours, forming highly crystalline COF membrane for the dye NF process. The high reaction rate and static solid phase of monomer can help overcome the mismatch between polymerization and crystallization of COFs, resulting in lowered membrane thickness in shorter time.

In other IP process of COFs, the monomers and catalyst were dissolved in oil and water, respectively, and the reaction happened at the oil-water interface when monomers reached the catalyst. $^{12}$ This monomers/catalyst manner was also adapted to the polymerization process at the air-liquid interface (after the evaporation of one solvent) by Lai in 2018. ${ }^{98}$ The monomers were dissolved in toluene, and then spread on the surface of water. After the evaporation of toluene, the polymerization of monomers at the interface was initiated by adding trifluoroacetic acid into the water, resulting in ultrathin COF membranes for dye NF process. The traditional IP process was applied to prepare COF membranes on the polysulfone (PSF) ultrafiltration (UF) membrane by Wang. ${ }^{100}$ The synthesis conditions (monomer concentrations and reaction time) were optimized to achieve continuous and permselective membranes. 1,4-phenylenediamine ( $\mathrm{Pa}$ ) and $\mathrm{Tp}$ were dissolved in water and $n$-hexane, respectively and then poured onto the PSF substrate step by step. The Pa solution was kept on the PSF surface for $0.5 \mathrm{~min}$ then drained off. After the addition of Tp solution, the color of the support changed to light yellow, indicating the forming of the COF membrane. This strategy considerably shortens the reaction time and can be considered as a promising technology for scalable preparation of COF membranes. In summary, optimization of the IP process toward preparation of MOFs or COFs using solvothermal methods would require careful selection of solvents, concentrations, operation conditions, etc. Furthermore, the control of interfacial polymerization via the surface modification of substrates can be applied and studied in the future to lower the membrane thickness to sub-10 $\mathrm{nm}, 116$ to realize the ultrafast transport of molecules in the separation process.

\subsection{Solution-casting approach}

The solution casting is usually applied to fabricate dense polymer membrane at laboratory scale. ${ }^{117}$ This method was scaled up towards membranes for medical applications, battery separators, and for special uses. In the typical solution-casting
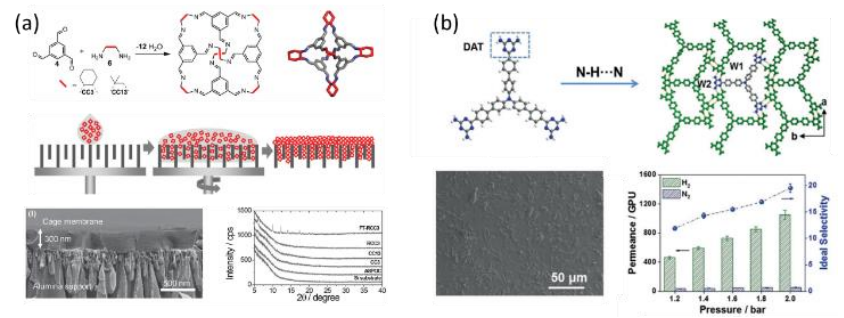

Fig. 3 Solution-casting processing approach for preparation of (a) POC and (b) HOF membranes. (adapted with permission from refs. ${ }^{103,104}$ ) 
process, the solid raw materials are dissolved in organic solvents with preferred moderate viscosity and volatility. The polymer solution then is spread across a flat surface with a casting knife. After the full evaporation of the solvent, a dense and flat membrane is formed. For the CPMs, their solubility is of crucial importance, and porous substrates can control the brittleness of the crystalline materials in the membranes. Zeolite crystals and COFs constructed by covalent bonds are basically insoluble in solvents. MOFs are easy dissolved in suitable solvents but with the broken of coordination bonds, and cannot be linked back after the removal of solvents. On the other hand, MOFs are in general not dispersible as the crystalline porous frameworks. However, in a recent work reported by Gascon, ZIF-67 particles with the outer surface functionalization can be stable dispersed in comparatively large solvent molecules such as cyclohexane. ${ }^{118}$ The resulting porous liquids that can be processed with polymers to form highly lorded MMMs for efficient propylene/propane separation. This outer surface functionalization strategies can be extended to other CPMs. With the assistant of crosslinkers or secondary growth process to seal the intercrystalline voids, the porous liquid with stable MOF dispersions can be applied for the scalable fabrication of continuous MOF membranes.

The POCs, a kind of molecules with intrinsic pores, can be dissolved in common solvents, ${ }^{109}, 119$ thanks to the lack of intermolecular covalent bond in the porous molecular assemblies. ${ }^{109}$ As reported by Cooper in 2016, a series of POCs produced by reactions between 1,3,5-triformylbenzene (TFB) with diamines were made into continuous membranes by solution-casting processing approach (Fig. 3a). ${ }^{103}$ The cages were firstly dissolved in chloroform or dichloromethane, then deposited into thin film on glass substrates by spin-coating. By adjusting the concentration of cages, various thickness of the membranes from $100 \mathrm{~nm}$ to $1 \mu \mathrm{m}$ were obtained. Furthermore, the morphology of cage films can be tuned by changing the composition of solutions, and by addition of methanol as a cosolvent. It should be noticed that using a cage solution with excess of methanol ( $>10 \mathrm{wt} \%$ ) resulted in hierarchically porous films with interconnected nanoparticles with a size of $\sim 100 \mathrm{~nm}$ due to the phase separation induced by solvent evaporation. This simple and generic approach was also applied towards preparation of continuous TFC membrane by depositing high concentrated cage solution on porous anodized aluminum oxide (AAO) filter disc. The obtained membranes showed comparable gas separation performance with other CPMBs, ${ }^{120}$ 124 while tended to densify over time, as reflected by loss in gas permeability. The author proposed potential solutions to construct more stable cage membranes via transforming cage thin films to more chemically stable ones, or introducing stronger intermolecular forces in the cages to form rigid and stable networks. Another important feature of these cage membranes is their amorphous nature without long-range order. This implies that the inter-molecular spaces formed are not uniform, although they have small sizes. On the other hand, the non-expanding feature of these porous cages can be combined with a modular 'mix-and-match' strategies. ${ }^{125,}{ }^{126}$ The "Ionely" cages with high separation property can be processed into "organic alloy" membrane with the assistance of the "sociable" cages with similar structure by a modular cocrystallization approach.

The extended HOFs structures can assemble into continuous membranes by solution-casting processing technology, due to the high reversible hydrogen bonds. ${ }^{16,}{ }^{111}$ In our recent work, the first HOF membrane was prepared on a common porous $\mathrm{Al}_{2} \mathrm{O}_{3}$ support (Fig. $3 \mathrm{~b}$ ). ${ }^{113}$ It was found that the mass nucleation and moderate growth rate were critical to fabricate a continuous HOF membrane. The highly concentrated HOF solution was coated on the support, then the solvent was evaporated at $80^{\circ} \mathrm{C}$ to form HOF membrane; this methodology can also be adapted to other supports such as AAO, PSF and $\mathrm{Si}$ wafers. Identical with the POCs, huge and isolated crystals were formed on the supports surface, when poor solvent was added to the casting solution. The HOF membrane exhibited pressureresponsive $\mathrm{H}_{2} / \mathrm{N}_{2}$ permeation that can be useful for separation of multiple mixtures. The reversible nature of linking bonds endowed the healing property of HOFs; the membrane can recover its separation performance after treatment with a vapor of solvents. Due to the weak hydrogen bonds, the stability of HOF membranes should be considered. In the most recent work by Farha, the 2D HOF structure was shown to be stable under harsh conditions (phosphate buffer solution and concentrated acid/base), with the help of the strong $\pi-\pi$ interaction. ${ }^{127}$ The protection coating applied in the cases of MOF membranes can also be used to enhance the stability of HOF and POC membranes. ${ }^{67,}$ 128-130

\subsection{Melt extruding approach}

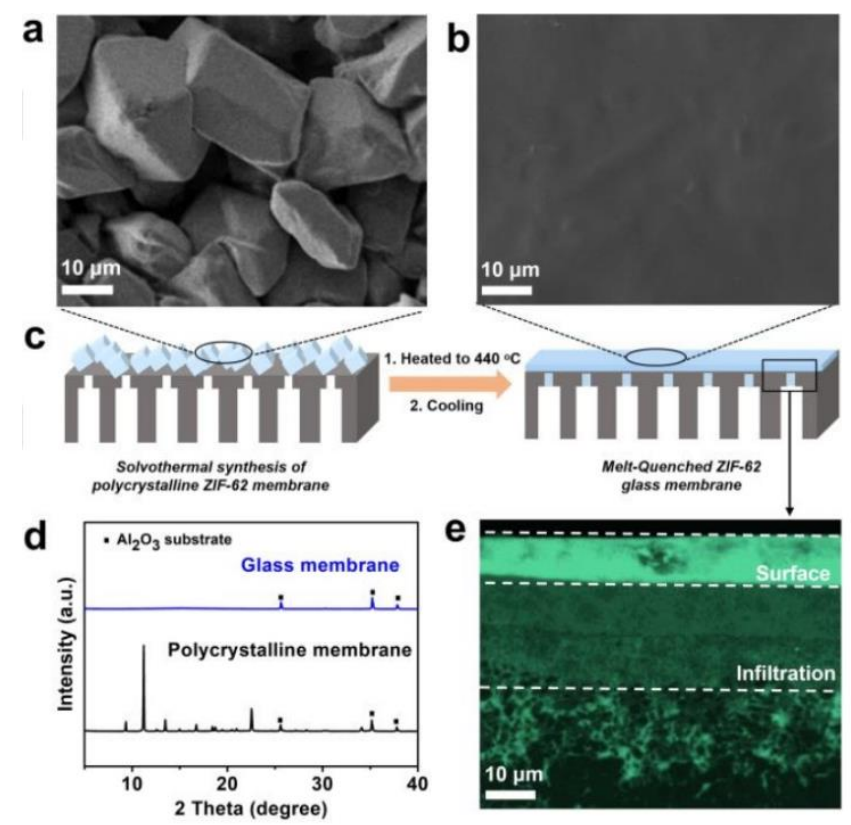

Fig. 4 Top SEM images of (a) polycrystalline ZIF-62 membrane and (b) ZIF-62 glass membrane. (c) Representation of the glass membrane formation process. (d) XRD patterns of glass and polycrystalline membranes. (e) EDXS-mapping of ZIF-62 glass membrane. (adapted with permission from ref. 63) 
(a)

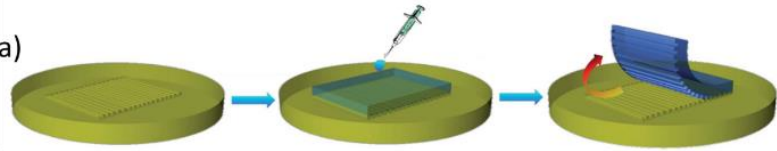

(b)

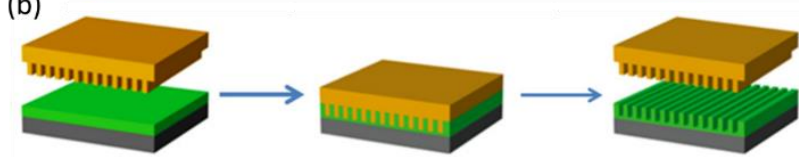

Fig. 5 Membrane surface patterning techniques: (a) solution casting micro-molding, (b) thermal embossing micro-molding. (adapted with permission from ref. ${ }^{131,132}$ )

The insoluble polymers at room temperature are not applicable for solution-casting technology, and therefore they are processed into membranes by melt extruding. The polymers are firstly melted by heating then pressed into thin film. ${ }^{133}$ This process is commonly used on a very large scale to make dense films for packaging applications. Most CPMs are not appropriate as the structures will be destroyed by the combustion or rearrangement of the organic ligands, before the materials are melted. However, several MOFs structures with low melting point $\left(T_{m}\right)$ were transformed into liquid before the ligands are changed ( $T_{m}<T_{d}, T_{d}$ is the temperature of decomposition). ${ }^{134-136}$ Nevertheless, the fully ordered structure is sacrificed during the cooling stage of the melted liquid, resulting in MOF glass. ${ }^{137,138}$ This MOF glass can be recognized as a subset of coordination polymers, which are very well reviewed by Bennett and Satoshi. ${ }^{135}$ For example the ZIF-4 $\mathrm{Zn}^{2+}$ linked with four imidazolate in a cag topology), the melting process is driven by the rare occurrence of $\mathrm{Zn}$-imidazolate bond dissociation at high temperature. ${ }^{139}$ After cooling, $\mathrm{Zn}^{2+}$ is also coordinated to four imidazolate ligands but, unlike the crystals, forms a continuous random network topology similar to that of amorphous $\mathrm{SiO}_{2}$. Besides heat treatment, other processes such as ball-milling and static pressure were also carried out for MOFs, followed with re-melting and cooling into bulk morphologies. Although the crystalline state is sacrificed, the narrow pore size distribution and selective adsorption property of the MOF glass can still be effective in separation.

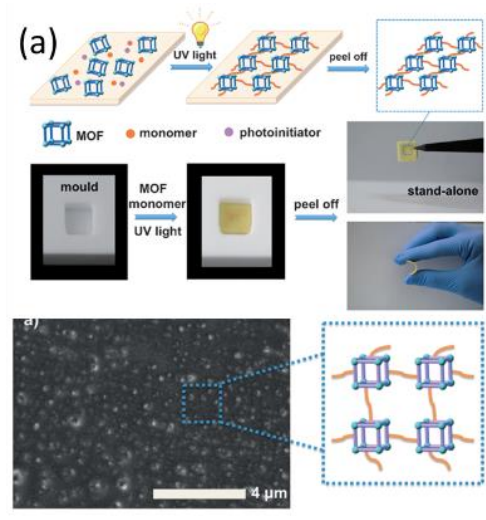

(b)
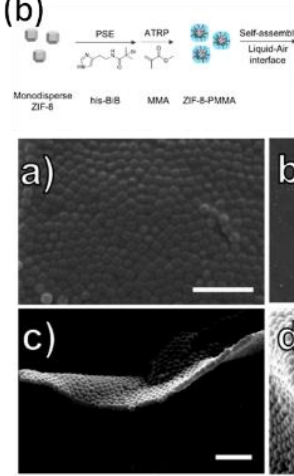

Recent work was reported by Jiang and Li, on the ZIF-62 $\mathrm{Zn}^{2+}$ is coordinated to imidazole and benzimidazole) glass membrane prepared for small gas separation (Fig. 4). ${ }^{63}$ The ZIF-62 crystals were firstly in-situ grown on the surface of porous ceramic alumina, followed by melt-quenching treatment. The molten ZIF-62 phase penetrated into the nanopores of the support and eliminated the formation of inter-crystalline defects in the resultant glass membrane. Positron annihilation lifetime spectroscopy was applied for mapping the pore size distribution of the ZIF-62 glass ( $3.16 \AA$ ), which was suitable for $\mathrm{H}_{2}$ separation from other large molecules. For this strategy, the dispersion of the precursor of ZIF- 62 crystals has to be controlled in order to obtain continuous membranes; the process requires optimization in order to achieve reversible transition. This approach and solution-casting will provide opportunities for shaping and tailoring of MOF glass membranes by the membrane surface patterning techniques such as solution casting micro-molding and thermal embossing micro-molding (Fig. 5). ${ }^{131}, 132,140,141$ The enhanced membrane surface area will be beneficial for their permeance, mitigating the impact of lower packing density of the classical CPMBs. Furthermore, these MOFs were blended with polymers, then melted and transferred into MOF glass based MMMs. Focus ion beam scanning electron microscopy, thermal characterization and membrane separation tests demonstrated the filling of voids at the MOF/polymer interface from the liquid MOF phase. ${ }^{137}$

Aside from MOF glass, the heat or press induced CPMBs preparation starting with raw materials but not from constructed frameworks were reported. ${ }^{22}, 142$ The solvent-free hot-pressing (HoP) method was utilized by Wang to prepare MOF film on a substrate, which can be utilize for roll-to-roll processing, which is considered as a mass production technique for MOF coatings. ${ }^{143,} 144$ The HoP method has some limitations for membrane separation that has to be addressed in the future, such as the voids between crystals and the applicability for high-valent MOFs. Moreover, the salt-mediated agent (PTSA) used in the IP preparation can also be added into mixtures of two ligands of imine based COFs to form freestanding COF membranes by heating and annealing for dye and nanoparticles filtration. ${ }^{101,} 145$ Due to the weak intermolecular interaction,
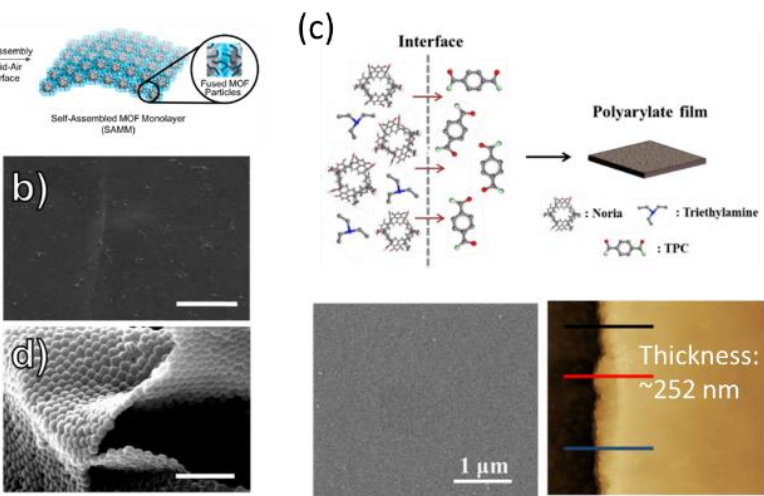

Fig. 6 Polymer-CPMs composite: (a) photoinduced post-synthetic polymerization of monomer functioned MOF; (b) self-assembly of PMMA modified MOF monolayers and freestanding multilayers; (c) IP of POC and monomers on a support into TFC membrane. (adapted with permission from ref. ${ }^{76,105,146)}$ 
both the HOF and POC membranes have potential to be process by melt extruding, while the reversible assembly of ordered structures is a challenge similar to the MOF glass.

\subsection{Polymerization approach}

Polymerization of CPMs with polymer is revealed as a visible approach to form hybrid materials. "PolyMOFs" were prepared using the polymerization approach. ${ }^{76}$ Cohen reviewed these materials in a recent paper. ${ }^{65}$ Here we will briefly introduce the method and our viewpoint on the scalable production of membranes using the polymerization approach. The MOF particles were modified with monomers, fragments or polymer brush, to make the MOF and polymer components more compatible and limit the formation of macrovoids at the interface (Fig. 6a-b). ${ }^{76,146-148}$ The MOFs were covalently bonded to polymers by photopolymerization, ${ }^{76,92}$ thiolene click, ${ }^{149}$ atom transfer radical polymerization, ${ }^{150}$ or ring opening metathesis polymerization. ${ }^{151}$ The obtained membrane (mostly mixed matrix membranes: MMMs) possessed improved separation performance for gas and liquid mixtures, especially improved permeability due to the high amount of MOFs in the composite materials. The interactions at the interface between the two components, i.e. the MOF and polymer were studied at the atomic level, 152 the focus was on understanding the formation of pores or the nonselective macro-voids. For practical application, thinner membranes for ideal flux and facile process are required. The interfacial polymerization of functional and ultra-small CPMs particles with common organic monomers should be a promising way to prepare thinner PolyMOFs.

Dispersed POCs with only one unit cell are suitable and can be polymerized to form continuous membrane. Recent reports by Niu described a POC: the Noria was polymerized with terephthaloyl chloride (TPC) into TFC membranes on polyacrylonitrile (PAN) UF membrane using the traditional IP technique (Fig. 6c). ${ }^{105}$ The POCs with an inherent high porosity were used as monomers and assembled in hybrid materials. Though it was not soluble in pure water, the Noria was dissolved in aqueous triethylamine solution. The phenolic hydroxyl groups of Noria were deprotonated after the addition of triethylamine, resulting in the formation of reactive phenoxide ions. The deprotonated Noria reacted with TPC through nucleophilic substitution and then polymerized into polyarylate. The inner cavity of Noria provided paths for the transport of both polar and non-polar solvents, which efficiently promoted the membrane permeance. In addition to the excellent performance, the simple preparation process with low-cost materials suggests the great potential of this type of TFC membranes for practical application.

The ultra-small CPM particles can play the role of monomer, just like the ethylene for polyethylene, polymerized in bulk samples. As reported recently, bulk $\mathrm{CaCO}_{3}$ materials were polymerized by $\left(\mathrm{CaCO}_{3}\right)_{\mathrm{n}}$ oligomers, ${ }^{153}$ which were stabilized with the trimethylamine as a capping agent (Fig. 7a). It should be noticed that the capping agents are also usually applied to synthesize nanosized MOFs, 154,155 which may provide opportunities for their assembly into continuous membranes. For the COFs,
Banerjee reported similar method, i.e., solution-processable imine based COF nanospheres were covalently self-assembled into thin film at the liquid-liquid interface (Fig. 7b), then transferred to the hollow fiber supports for single gas ( $\mathrm{He}, \mathrm{N}_{2}$, $\mathrm{O}_{2}, \mathrm{CO}_{2}$, and $\mathrm{CH}_{4}$ ) permeation tests. ${ }^{102} \mathrm{~A}$ direct nucleation approach (diluted mother solution with PTSA) was introduced to avoid the undesired precipitation of the amorphous polymer during the formation of the crystalline COF nanospheres with a size in the range from 25 to $570 \mathrm{~nm}$. The presence of free amine and aldehyde functional groups in the COF nanosphere was confirmed by FTIR. The covalent bonding at the waterdichloromethane interface resulted in a continuous COF thin film. This method can be adapted towards preparation of TFC membrane on UF supports.
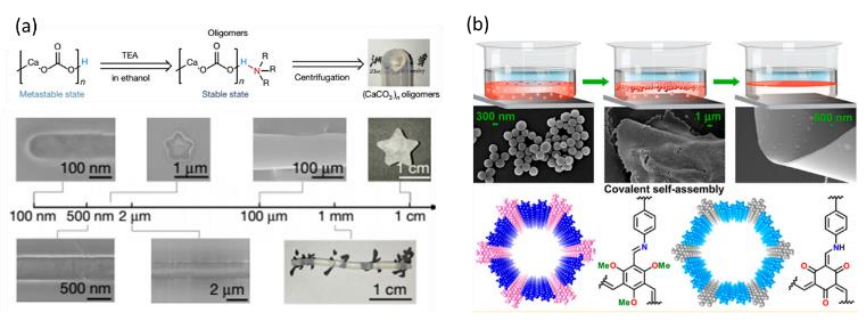

Fig. 7 Polymerization of active oligomers on (a) $\mathrm{CaCO}_{3}$ and (b) imine-typed COFs. (adapted with permission from ref. ${ }^{102,153}$ )

\section{Strategies tailored based on the properties of CPMs}

Compared with polymers, CPMs possess crystalline structures, and they can be assembled in membranes by an epitaxial growth on pre-dispersed seed crystals; this approach is revealed as seeding-secondary growth process. ${ }^{21}, 43,156$ Using the seeding-secondary growth, zeolite membranes have been scale-up and practically applied for the removal of water from organic solvents. ${ }^{157}$ CPMs containing metal species were used for precursor conversion, ${ }^{82,158}$ electrochemical and CVD scaleup deposition approaches. ${ }^{59,}$ 94, 159 In this section, we will review these methods applied for preparation of CPMBs, and the development and challenging issues will be summarized.

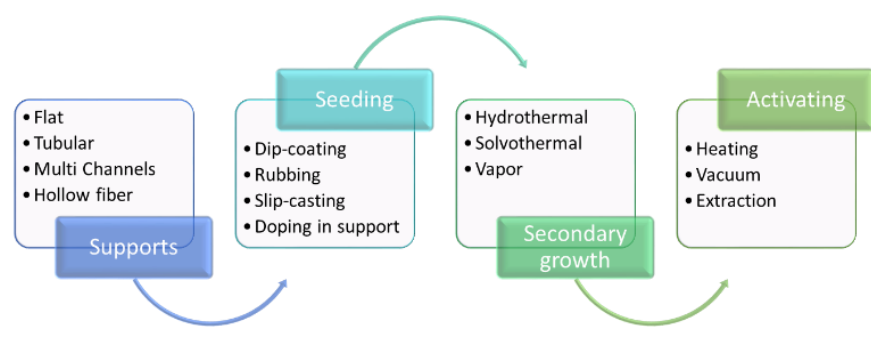

Fig. 8 Stages of seeding-secondary growth of CPMBs on supports, from left to right: selection of supports, different methods for seeding, secondary growth and activation of membranes. 


\subsection{Seeding-secondary growth approach}

The seeding-secondary growth approach was firstly applied for the preparation of continuous pure zeolite membranes. Zeolites are porous crystalline aluminosilicates with well-defined channel structures of molecular dimensions. ${ }^{160}$ Before the seeding-secondary growth method was developed, the in situ hydrothermal synthesis approach for preparation of polycrystalline zeolite membranes was widely used. The porous substrates $\left(\mathrm{Al}_{2} \mathrm{O}_{3}\right.$ or stainless steel substrates) with or without pre-treatments were immersed in mother suspensions or gels sealed in autoclaves ( $\mathrm{Al}, \mathrm{Si}$ and other source) and subjected to hydrothermal treatment at the suitable conditions $(\mathrm{pH}$ value, concentration, temperature, etc.). The oxide layers of the supports combined with the high roughness enriched of defects was shown to be beneficial by improving the interactions between the crystals in the membranes. However, large voids between the zeolite crystallites were formed, which is the main challenge faced in the preparation of polycrystalline membrane. ${ }^{45}$ Using organic structural directing agents and optimizing the reaction conditions, defect-free membranes by in-situ growth were obtained but with low reproducibility. In order to improve the continuity of the membranes, the supports were immersed in the mother solutions for a second time; this process was described as a seeding-secondary growth. Using the seeding-secondary growth continuous zeolite membranes within a wider variations of reaction conditions were obtained. Usually, small-sized zeolites were used as seed crystals $(<1 \mu \mathrm{m})$, then deposited on the support surface, followed by additional crystal growth process (Fig. 8). ${ }^{161}$ The even dispersion and anchor of zeolite seeds on the substrates is of great importance to realize the continuous membrane. The different seeding techniques such as dip-coating, slip-casting, vacuum-coating, spin-coating, rubbing the support have been widely used to prepare zeolite membrane with high quality, ${ }^{47}$, 162-169 among which dip-coating is the most widely used method, especially for zeolite membranes on tubular supports. To stabilize the seeds on the substrates, a heating treatment was always combined with dip-coating process. Then the excess of seeds from the substrates was removed by wiping thus ensuring clean and full coverage of the membrane. ${ }^{47,170}$ To improve the mechanical stability of seed layers, some additives were introduced in the seed suspension, such as hydroxyl propyl cellulose, polyethyleneimine and colloidal silica. ${ }^{171-173}$

In 1999, the first industrial facility for ethanol dehydration using $\mathrm{NaA}$ zeolite membranes was established by Mitsui Engineering and Shipbuilding Co., Japan. ${ }^{39}$ This LTA type zeolite membrane with $20-30 \mu \mathrm{m}$ thickness was grown on a tubular support by the seeding-secondary process (125 tubes were used in the construction of a module). Similar technology was used for preparation of modules of LTA zeolite for water removal by several companies in Germany, England and China. ${ }^{174-176}$ To further increase the packing density, hollow fibers were used as supports as well. ${ }^{177}$ Wang reported a dip-coating-wiping seeding method to obtain LTA membranes with high water/ethanol separation performance and high reproducibility on ceramic hollow fiber supports. Dip-coating produces a mostly uniform seed layer, but there are still gaps that result in poor coverage of zeolite membranes. The wiping step makes seeds become more uniform and possibly enter into the defects on the surface of the support, leading to the high-quality dense membrane. In order to reduce the cost of supports, zeolite/polymer hybrid materials were used as substrates. ${ }^{178}$ Besides the ceramics and hybrid hollow fiber supports, stainless steel (SS) hollow fiber is also believed to be promising for industrial application due to its robustness. Besides that, the porous SS hollow fiber substrates have relatively large pores and high surface roughness, which are favorable for the formation of zeolite membrane. Some linkers (poly(ethyleneimine), ${ }^{179} \quad 3$-aminopropyltriethoxysilane, ${ }^{180}$ polydopamine, ${ }^{181}$ diisocyanates, ${ }^{182}$ thionyl chloride, ${ }^{183}$ carboxymethyl chitosan ${ }^{184}$ and polyvinyl pyrrolidone ${ }^{185}$ ) were used to modify the SS hollow fiber surface so as to increase the interactions between seed crystals and supports. Microwave (MW) heating was applied by Yang to decrease the membrane thickness. ${ }^{186}$ Under the microwave irradiation, the zeolite crystal growth rate was increased substantially resulting in the formation of defect free membranes with low impurities. ${ }^{51,187}$ Compared to the conventional heating, the MW resulted in low overall membrane thickness that facilitated the species pathways at the interface of the zeolite and the substrate. Up to now, several types of zeolites (LTA, FAU, CHA and MFI type structures) have been successfully used for preparation of membrane modules via seeding-secondary growth method, ${ }^{39}$, $172,188,189$ thus proving the universality of this strategy.

In addition to the facile preparation conditions, the seedingsecondary growth method also allows the preparation of thin and oriented crystalline membrane for highly efficient separation. Excellent papers have been reported by Tsapatsis and Yoon. ${ }^{52,53}$ However the scalable production of this oriented zeolite membranes is still challenging. Yoon reported the gelless secondary growth procedure in which secondary growth was performed in a sealed autoclave with only a small amount of an aqueous SDA solution impregnated in the supported seed layer. ${ }^{54}$ In addition to cost savings, this method is expected to be easily scale-up with high reproducibility since it avoids the need to control heat and mass transfer in a complex reactive liquid to achieve in-plane growth of oriented seeds. Furthermore, Yoon and Tsapatsis reported on zeolite nanosheet seeding and gel-less secondary growth as a scalable, robust, and reproducible method to prepare intergrown and oriented MFI films as thin as $100 \mathrm{~nm}$ on porous Stöber silica supports for isomer mixture separation. ${ }^{55}$

Another approach is the steam-assisted conversion as a powerful technology towards production of oriented zeolite membranes will be discussed in section 4.2. The last point of consideration when dealing with zeolite membranes is the calcination that is required to remove the organic templates used for the synthesis of some zeolites. This requires a final activation at elevated temperature (above $400{ }^{\circ} \mathrm{C}$ ) that may lead to the formation of cracks and defects, especially in the case of small-pore zeolite membranes. Thus, the organic template-free condition to produce nanosized zeolite particles is highly desirable for seeding-secondary growth of thin zeolite membranes. Mintova group has reported the syntheses of 

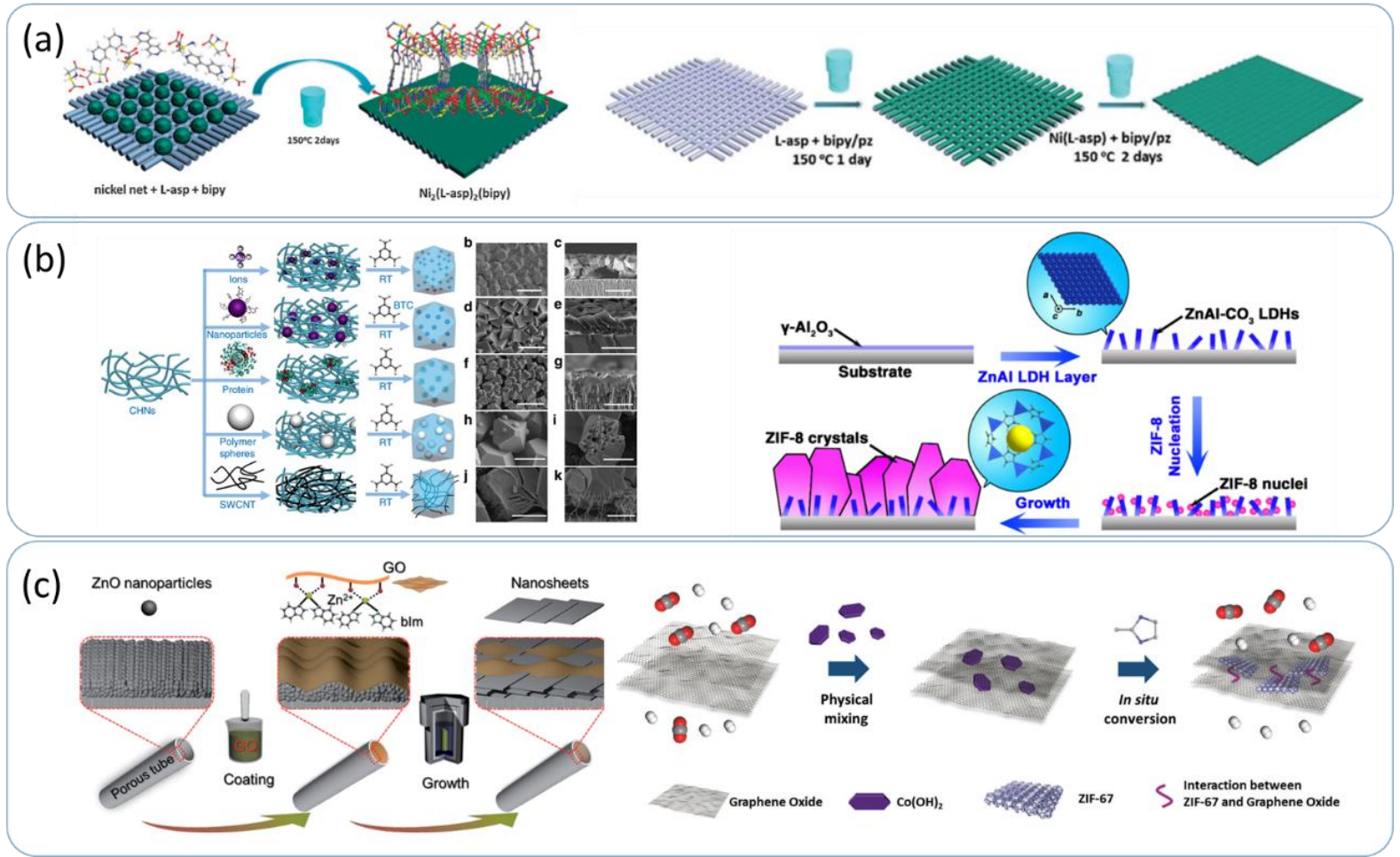

Fig. 9 Preparation of MOF membranes using the conversion of precursors on: (a) metal- net substrate, (b) metal-dense substrate, and (c) confinement transformation between 2D materials. (adapted with permission from ref. $64,84,85,88,190,191$ )

several template free nanocrystals with SOD, FAU, RHO, CHA and EMT framework structures. ${ }^{160,192-194}$

The seeding-secondary growth method of zeolite membranes (seed dispersion, support modification and optimization strategy of mother solution) can be transferred to the preparation of MOF membranes as well. Compared with zeolite, the post-treatment step is easier for MOFs, the pores of most MOFs can be activated at mild condition (temperature below $100{ }^{\circ} \mathrm{C}$ ). Moreover, based on the features of MOFs, several novel processes are applied to coat the uniform seed layers on supports, ${ }^{78}$ such as reactive seeding method, ${ }^{195}$ step-by-step deposition $^{196}$ and precursor conversion. ${ }^{190}$ However, the obstacles for the scale-up of MOF membranes by seedingsecondary growth are similar to those of zeolites, such as the extended long reaction time, large amount solvents consumption, thick membrane and expensive supports. Some of which can be overcome by changing the production method, while solving all the problems is still difficult. Another issue is the low stability of most MOF structures, which is needed to be improved prior the practical applications.

\subsection{Conversion of precursors into crystalline membranes}

As it is challenging to grow continuous polycrystalline membrane directly, the amorphous precursors are firstly coated on a support, then transformed to the CPMBs by post treatment. This method has been applied to prepare zeolite and MOF membranes. For the zeolite membrane, the dry gel containing the "nutrients" and with or without SDAs are coated on a support then treated under steaming without or with SDAs to convert into crystalline membranes; ${ }^{197}$ this method is called a dry-gel conversion. Xu and Dong reported the conversion of a dry aluminosilicate gel into MFI type zeolite and film by putting the gel in contact with water and amine vapors. ${ }^{198,} 199$ The SDA was not included in the dry precursor gel but in the liquid phase, thus the methods is referred to a vapor phase transformation. A similar method was applied by Matsukata to produce ZSM-5, ferrierite, mordenite and analcime membranes on flat porous supports for $\mathrm{O}_{2} / \mathrm{N}_{2}$ separation. ${ }^{56,} 200$ Tubular membranes are preferable to flat ones on account of their simpler implementation in industrial scale production. In the study of Santamaria, ${ }^{57} \mathrm{MFI}$ type zeolite membranes were successfully prepared on both alumina and stainless steel tubular supports by additional crystallization of initially deposited silicate layers under steaming. The dry gel contained the organic template agents while the vapor phase is a steam without SDAs, and this method is referred to the steam-assisted crystallization. In the work reported by Cui, a high-strength self-supporting tubular LTA zeolite membrane was prepared by in situ hydrothermal transformation of a geopolymer membrane. ${ }^{201}$ The surface and inner amorphous geopolymer membrane was converted into zeolite crystalline phase after the hydrothermal process, and the self-standing membrane possessed the good mechanical strength under the high pressure (57 $\mathrm{MPa}$ ); these membranes were used for desalination. Nair fabricated high-silica CHA zeolite membranes on low-cost alumina hollow fibers by a onestep direct gel conversion method for $\mathrm{CO}_{2}$ separation. ${ }^{44}$ The 
precursor mixture contained both a continuous gel phase as well as pre-synthesized CHA submicron crystals to promote crystallization. It was shown that the gel composite, conversion condition and time are of great importance for the preparation of high-quality membranes. Compared with the tradition hydrothermal process, the dry gel conversion method not only leads to homogeneous nucleation and growth of crystals that could improve the quality of the zeolite membrane, but also has the additional advantage of minimizing the waste reactants. Moreover, in the case of pre-oriented seeds, the gel-free method can prevent the dissolution and random regrowth of oriented seeds that usually occurred during the hydrothermal treatment, leading to oriented zeolite membranes. ${ }^{202}$

The precursors for MOF membranes usually are metal based materials, such as pure metals, metal oxides, hydroxides and gels. Qiu reported a series of papers on the growth of $\mathrm{Cu}, \mathrm{Zn}$ and $\mathrm{Ni}$ based MOFs on the metal substrates (Cu mesh, ${ }^{203} \mathrm{Zn}$ wafer ${ }^{204}$ and $\left.\mathrm{Ni} \mathrm{mesh}^{8}\right)$. The supports were immersed in solutions containing organic ligands at the solvothermal condition that promoted the metal ions to release from the supports and reacted with ligands thus forming MOF membranes (Fig. 9a). Then Peng applied metal hydroxide nano-strands (copper MHNs) as a precursor in 1,3,5-benzenetricarboxylic acid (BTC)water-ethanol solution at room temperature for the preparation of MOF membranes. ${ }^{79}$ This approach can be applied for the large scale production, it is robust and as a result well-intergrown free-standing HKUST-1 membranes for gas separation $\left(\mathrm{H}_{2} / \mathrm{CO}_{2}\right)$ were constructed. The truncated crystals were controllable and favorable for the dense intergrowth processing. In their further work, various dispersions of functional components (ions, nanoparticles, polystyrene spheres, single-walled carbon nanotubes or bioactive proteins) were mixed with the MHNs solution, then transformed to the HKUST-1 thin film containing all functional components (Fig. 9b). ${ }^{191}$ The same precursor was also vacuum loaded on the outer surface of Poly(vinylidene fluoride) (PVDF) hollow fiber supports to produce MOF membranes. ${ }^{80}$ The universality of this approach towards preparation of membranes on various supports at mild conversion condition was demonstrated. Another advantage of this strategy is that the embedded species can also affect the orientation of grown crystals and the separation performance of the membranes. ${ }^{205}$ This method was adapted for the preparation of ZIF-8 form a precursor of zinc MHNs. ${ }^{206}$ Zhang and Li et al. fabricated highly active $\mathrm{ZnO}$ buffer layers on PVDF hollow fiber, then converted into uniform ZIF layers by treatment with a 2-MIM solution for $\mathrm{H}_{2}$ separation. ${ }^{207}$ The layered double hydroxides (LDHs) were also applied as a precursor to form continuous MOF membranes. As reported by Liu and Caro, the $\mathrm{LDHs}$ of $\mathrm{ZnAl}-\mathrm{CO}_{3}$ or $\mathrm{ZnAl}-\mathrm{NO}_{3}$ were firstly modified on the porous $\alpha-\mathrm{Al}_{2} \mathrm{O}_{3}$ substrates, which promoted the heterogeneous nucleation of ZIF-8 crystals. $^{83, ~ 84, ~} 208$ After the solvothermal reaction in a 2-MIM-containing methanol solution, the part of LDHs was transformed and resulted in the LDH-ZIF-8 composite membrane with favored permeation for $\mathrm{H}_{2}$. The metal gel or atomic layer deposition (ALD) induced $\mathrm{ZnO}$ layers also played the role of precursor, and were converted to ZIF-8 by vapor treatment (see section 4.4). ${ }^{94}$
The scalable preparation of highly oriented and ultrathin MOF membranes especially on standard tubular supports remains a challenge. Zhang used 2D material of graphene oxide (GO) to direct the growth of MOF membrane (Fig. 9c). ${ }^{85} \mathrm{~A}$ thin layer of ZnO NPs was confined between a substrate and a GO ultrathin layer and self-converted under the assistance of ligands into a highly oriented $\mathrm{Zn}_{2}(\mathrm{blm})_{4}$ nanosheets membrane for $\mathrm{H}_{2}$ separation. The confinement transformation strategy is also successful in the fabrication of composite membrane based on nanosized MOF and GO. ${ }^{87,} 209$ The metal based nanosheets as precursor were filtrated into the GO precursor membranes by direct mixing or layer-by-layer method. ${ }^{88,} 210$ The metal nanosheets were transformed to MOFs under reaction with ligands at room temperature. The type of metal nanosheets (oxide or hydroxide) and metal nanosheets/GO ratio are of significant importance for the quick and complete conversion to MOFs. 88 This method was applied towards preparation of zeolites as well; the metal nanosheets and ligand solution were replaced by an amorphous precursor gel and $\mathrm{NaOH}$ solution. ${ }^{58}$ Through the in-situ conversion process, the strong bonding between the CPMs and GO was achieved resulting in membranes with high selectivity for $\mathrm{H}_{2} / \mathrm{CO}_{2}$, avoiding the formation of non-selective interface void (Fig. 10). This type of composite membrane combines the molecular sieving of CPMs and the easy processing of GO material, which is a promising way for scalable production of efficient separation modules.
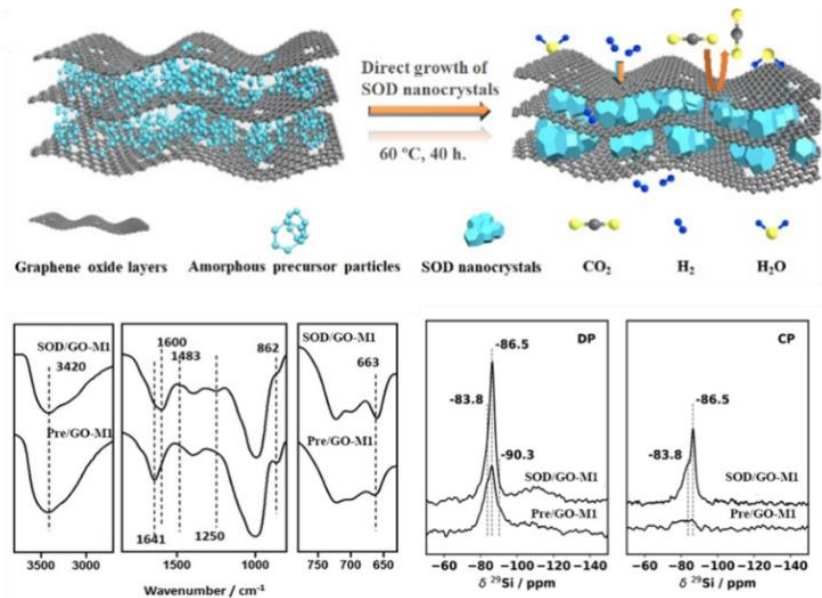

Fig. $10 \mathrm{SOD} / \mathrm{GO}$ composite membranes prepared by direct transformation of amorphous precursor particles infiltrated in GO matrix. The cross-linking of SOD nanoparticles with the $\mathrm{GO}$ in the membranes promoted the gas separation performance. (adapted with permission from ref. ${ }^{58}$ )

\subsection{Electrochemical preparation}

Electrochemical polymerization (EP) offers a simple and scalable way to prepare polymeric films within a short reaction time under mild conditions. ${ }^{211}$ Recently, the EP was applied to fabricate the scalable polymer membranes with inherent pores by adjusting the polymeric active sites and the alkyl chain length of the monomers for $\mathrm{H}_{2}$ separation. ${ }^{159}$ Although it is also a process of converting electrical energy into chemical energy, the electrochemical preparation of CPMBs is more adapting the 


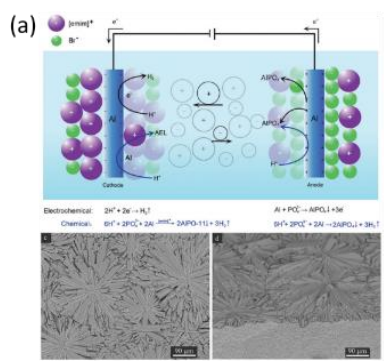

(b)

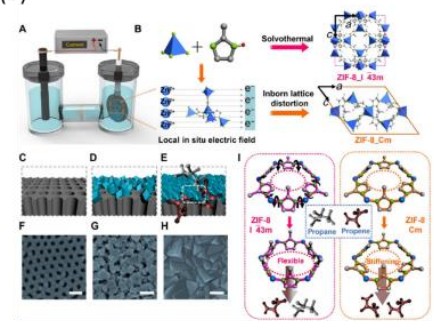

Fig. 11 Preparation of (a) zeolite and (b) MOF membranes by electrochemical deposition (ED). (adapted with permission from ref. ${ }^{90,212}$ )

technology from the electrodeposition (ED) of ceramic films, ${ }^{213}$ rather than the EP process. ED is a common and effective technique for preparation of thin films. In the ED process, charged particles migrate to the support surface homogenously under the action of the applied electric field, resulting in the formation of uniform, dense and thickness-controllable membranes in short time. As reported by Cazorla-Amoros, an external electric field can be applied to align zeolite crystals on substrates to form a seed layer for the secondary growth of polycrystalline zeolite films. ${ }^{214}$ This process must be followed by a hydrothermal step to obtain a fully dense molecular-sieve layer. Yang prepared the uniform LTA zeolite membranes on tubular porous $\alpha-\mathrm{Al}_{2} \mathrm{O}_{3}$ supports under the electric field for water/isopropanol separation. ${ }^{59}$ The charged zeolite particles were attracted and transported to the support resulting in homogeneous membrane. To overcome the narrow electrochemical window of water, the same group used ionic liquids (ILs) as a solvent for in situ electrochemical deposition of oriented and defect-free AEL $\left(\mathrm{AIPO}_{4}-11\right)$ zeolite layers (Fig. 11a). ${ }^{212}$ It should be noticed that the Al electrode served as an Al source as well, and the $\mathrm{AlPO}_{4}-11$ was only formed on the surface of cathode. At the negatively charged cathode, the [emim] ${ }^{+}$cations of the IL were electrostatically adsorbed and acted as a template (SDA) for $\mathrm{AlPO}_{4}-11$. Under the effect of the SDA, the cathode reacted with protons and converted the electrode surface to $\mathrm{AlPO}_{4}-11$ with $\mathrm{PO}_{4}{ }^{3-}$. The simple approach carried out under ambient pressure with controllable electrochemical parameters may have a great potential for a scale-up to industrial application.

The ED method is applied for preparation of continuous MOF films and membranes at room temperature for a short time and the conductive supports were mostly applied. Similar to zeolites, the electrode can provide a source of reagents (metal ions, if metal electrode is used) and promote reactions such as deprotonation of ligands and attraction of metal ions or deposition and growth of metal-ligand complexes into MOFs. An electrophoretic nuclei assembly for crystallization of highly intergrown thin-films was reported by Agrawal for propylene/propane separation. ${ }^{89}$ This approach was applied for ZIF-8 and ZIF-7 membranes on various substrates including porous polyacrylonitrile, anodized aluminum oxide, metal foil, porous carbon and graphene. By adjusting the applied electric field $(E)$ and alkalinity of solutions $(\mathrm{pH})$, the heterogeneous nucleation density was controlled resulting in a highly packed

layer (nuclei film) from a very dilute precursor sol. The high density of nuclei film facilitated the growth of highly packed intergrown crystals in the same precursor sol, and this process is similar to the classical seeding-secondary growth but more efficient. By performing the ED process at $30^{\circ} \mathrm{C}$ from $2 \mathrm{~h}$ to $8 \mathrm{~h}$, the defect-free ZIF-8 membranes were fabricated with a thickness from $176 \mathrm{~nm}$ to $375 \mathrm{~nm}$. Inspired by this work and the discovery that ZIF-8 transformed to more rigid lattices by applying an external electric field, ${ }^{215}$ Wang described a fast $20-$ min preparation procedure of ZIF-8 membranes with inbornsuppressed linker mobility by a fast current-driven synthesis (Fig. 11b). The ZIF-8 membranes were grown at a low direct current $\left(0.7 \mathrm{~mA} / \mathrm{cm}^{2}\right) .{ }^{90}$ An electrochemical cell was designed to grow ZIF-8 membrane with a double function of the external direct current. The applied method promoted the deprotonation of the linker 2-MIM to the imidazolate anion and simultaneously $\mathrm{Zn}^{2+}$ cations were attracted thus boosting the crystallization of ZIF-8 on the substrate. This gave rise to a fast membrane growth at room temperature, and just several minutes were enough for the formation of continuous membrane layer, showing great scalability potential. More importantly, the external direct current formed a local in situ electric field and caused inborn lattice distortion of the ZIF-8 structure. Therefore, membrane layers of distorted ZIF-8 polymorphs with rigid frameworks and long lifetime were prepared, with molecular sieving performance for $\mathrm{C}_{3} \mathrm{H}_{6} / \mathrm{C}_{3} \mathrm{H}_{8}$ mixtures. The same group applied this process to produce a mixed-linked ZIF-8 membrane with fine tuning of the aperture size. This optimized ZIF- $7_{22}-8$ membrane showed significant improvement in the $\mathrm{CO}_{2} / \mathrm{CH}_{4}$ molecular sieving process. ${ }^{91}$ Furthermore, they used similar method to prepare the bimetallic Zn(100-x)Cox-ZIF membrane, which attained the best balance between the framework flexibility and the grain boundary structure, and showed $\mathrm{C}_{3} \mathrm{H}_{6} / \mathrm{C}_{3} \mathrm{H}_{8}$ separation factor of 200 with excellent stability.

For the ED preparation of MOF membranes, organic solvents with the addition of electrolyte or modulator are usually applied, which result in a complex system and concerns for the potential negative impacts on the environment in the largescale industrial production. Addressed this point, Lai reported the fabrication of ZIF-8 membranes via aqueously cathodic deposition (ACD) without addition of any supporting electrolyte or modulator for propylene/propane separation. ${ }^{93}$ The fabrication process used $100 \%$ water as a single solvent and a low-defect membrane was obtained in 60 min only at room temperature without any pre-synthesis treatment. Anodized aluminum oxide (AAO) with a conductive coating as a working electrode was applied, while graphite paper without any pretreatment as a counter electrode was used. The $\mathrm{Zn}^{2+}$ attracted by the AAO cathode resulted in the assembly of zinc-ligand complexes, where the $\mathrm{OH}^{-}$released from the reduction of water facilitated the deprotonation of these complexes. These deprotonated complexes then further oligomerized, nucleated, crystallized, and finally formed a dense ZIF-8 layer on the AAO support. An optimal current density of $0.13 \mathrm{~mA} \mathrm{~cm}^{-2}$ was identified, which ensured both slow water electrolysis (avoiding 
corrosion of the already formed MOF by excessive $\mathrm{OH}^{-}$) and quick deposition rate. This $A C D$ approach with an ultrafacile set-

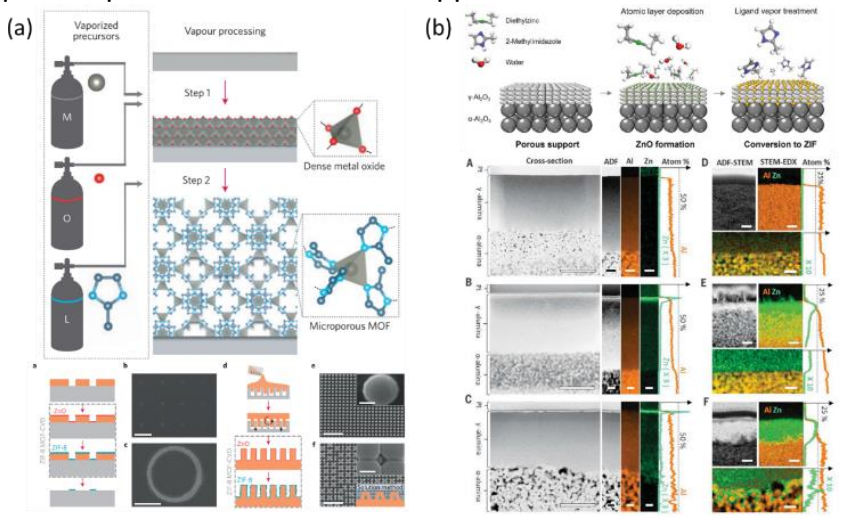

Fig. 12 Preparation of MOF (a) pattern thin film and (b) membrane by CVD method. (adapted with permission from ref. ${ }^{94,216}$ )

up is a promising method for scalable and environmentally friendly production of MOF membranes.

The ED offers a scalable and time-saving strategy to fabricate zeolite and MOF membranes. ${ }^{217}$ However, scale-up ED of CPMBs on large and low-cost conductive supports have to be further optimized. The relationship between the $E$ and framework structures should be further explored to prove the method's universality. Ordered COF membranes may be prepared by optimizing the condition of EP process in the future.

\subsection{Chemical vapor deposition}

The solvothermal or hydrothermal preparation processes usually consume large amount of solvents, and dissolved reactants are difficult to recovery and recycled. A more environmentally friendly method is required to expand the production of CPMBs. In 2016, CVD was applied by Ameloot to fabricate ZIF-8 film through an all-vapor process on Si wafer (Fig. 12a). ${ }^{216}$ The 'MOF-CVD' method consists of two steps: a metal oxide deposition step and a vapor-solid reaction step. Initially, the metal precursor (zinc oxide) with controlled thickness was coated onto the surface of $\mathrm{TiO}_{2}$ substrates by ALD, then was transformed to ZIF-8 by exposure to $2-\mathrm{MIM}$ vapor at $100{ }^{\circ} \mathrm{C}$ for $30 \mathrm{~min}$. Compared with the solvothermal preparation in solution, the formation of rough films, pinholes and even homogeneous nucleation of MOF particles are largely avoided by supplying the organic linker in a vapor phase. This approach enables the deposition of ZIF-8 thin films with a uniform and controlled thickness, even on supports with high-aspect-ratio features. The compatibility of MOF-CVD with existing and scalable fabrication infrastructure, both in research and production facilities, will greatly facilitate MOF integration in related applications. This method also shed light on the development of surface pattern MOF membranes.

A similar process was reported by Tsapatsis, ZIF-8 membrane on porous $\alpha$-Alumina substrate was prepared and used for propylene/propane separation (Fig 12b). ${ }^{94}$ An impermeable zinc oxide and/or zinc hydroxide were deposited on the top and inside the $\alpha$-alumina, then treated by 2-MIM vapor to transform into ZIF-8. Unlike other molecular sieve membrane fabrication methods which rely on solvothermal nucleation and growth that are difficult to be scaled up, this method is solvent-free and seed-free thus can be considered for scale up technology. The 2-MIM vapor can also be used as a secondary growth "solution" to seal the defect and provide ultra-thin ZIF-8 membranes. In 2017, Li and Zhang fabricated scalable ZIF-8 membranes on hollow fibers from the zinc gel by reacting with the 2-MIM vapor. ${ }^{82}$ By adjusting the sol concentration and the coating procedure, a nanometer-thick MOF membrane $(<20 \mathrm{~nm})$ was obtained for highly efficient $\mathrm{C}_{3} \mathrm{H}_{6} / \mathrm{C}_{3} \mathrm{H}_{8}$ separation. Combined with another idea of their previous works, ${ }^{218} \mathrm{Li}$ adapted this linker vaper-phase treatment to the linker change process of MOF powder materials, redesigning the pore environment to greatly improve $\mathrm{CO}_{2} / \mathrm{N}_{2}$ and $\mathrm{CH}_{4} / \mathrm{N}_{2}$ selectivity. ${ }^{219}$ The vapor phase transformation strategy has also been explored by Zhang to direct the synthesis of a 2D Co-based nanosheet MOF membrane on a porous tubular substrate via ligand vapor phase transformation. ${ }^{86}$ The gel layer is multifunctional, i.e., active metal source and guided the oriented growth of nanosheets and controlled the membrane thickness. The Co-based nanosheet membrane with a thickness of $57 \mathrm{~nm}$ exhibited excellent $\mathrm{H}_{2} / \mathrm{CO}_{2}$ separation performance. This method is considered as a promising for scalable fabrication of MOF nanosheet membranes.

However, similar to the EP process, only the ZIF series membranes now have been prepared by CVD method, mainly because the ligands of most MOFs are complex and not applicable for vapor phase deposition. Therefore, expanding of the CVD to more MOFs is another promising pathway to improve the separation performance of the membranes.

\section{Conclusion and outlook}

In the last decade, the CPMBs separation membranes become important for various environmental and energy-related processes. A great work has been devoted to the development of CPMBs. Diverse methods have been applied to synthesize various CPMs and deposit them on membrane modules. There are still challenges but also opportunities for the vast production of these membranes. Scalable fabrication of thin and continuous membranes with stable separation performance on cost acceptable supports is still the goal to achieve, all existing solutions still need to be optimized to meet all requirements. Up to now, Interfacial polymerization (IP) and seeding-secondary growth methods are in leading positions to reach the target because substantial research achievements were already reported. For the IP process, the rich experience, technology and supports of polymer membranes are readily to transfer to CPMBs, while the process conditions have to be readjusted toward crystallization of CPMs, balancing the order and continuity. Beyond ZIF- 8 and HKUST-1, more stable MOF structures such as UiO and MIL fabricated as membranes by IP are highly demanded.220, 221 The pre-treatment of building blocks like processed into "semi-finished products" may pave the way for preparation of scalable membranes. ${ }^{222}$ While in the seeding-secondary growth of zeolites, the excluding of SDA 
from the process is highly desirable in order to prevent the formation of defects caused by the final activation step. The self-healing process or the healing treatment with modifiers are

Table 6 Outlook of current strategies for scalable preparation of CPMBs

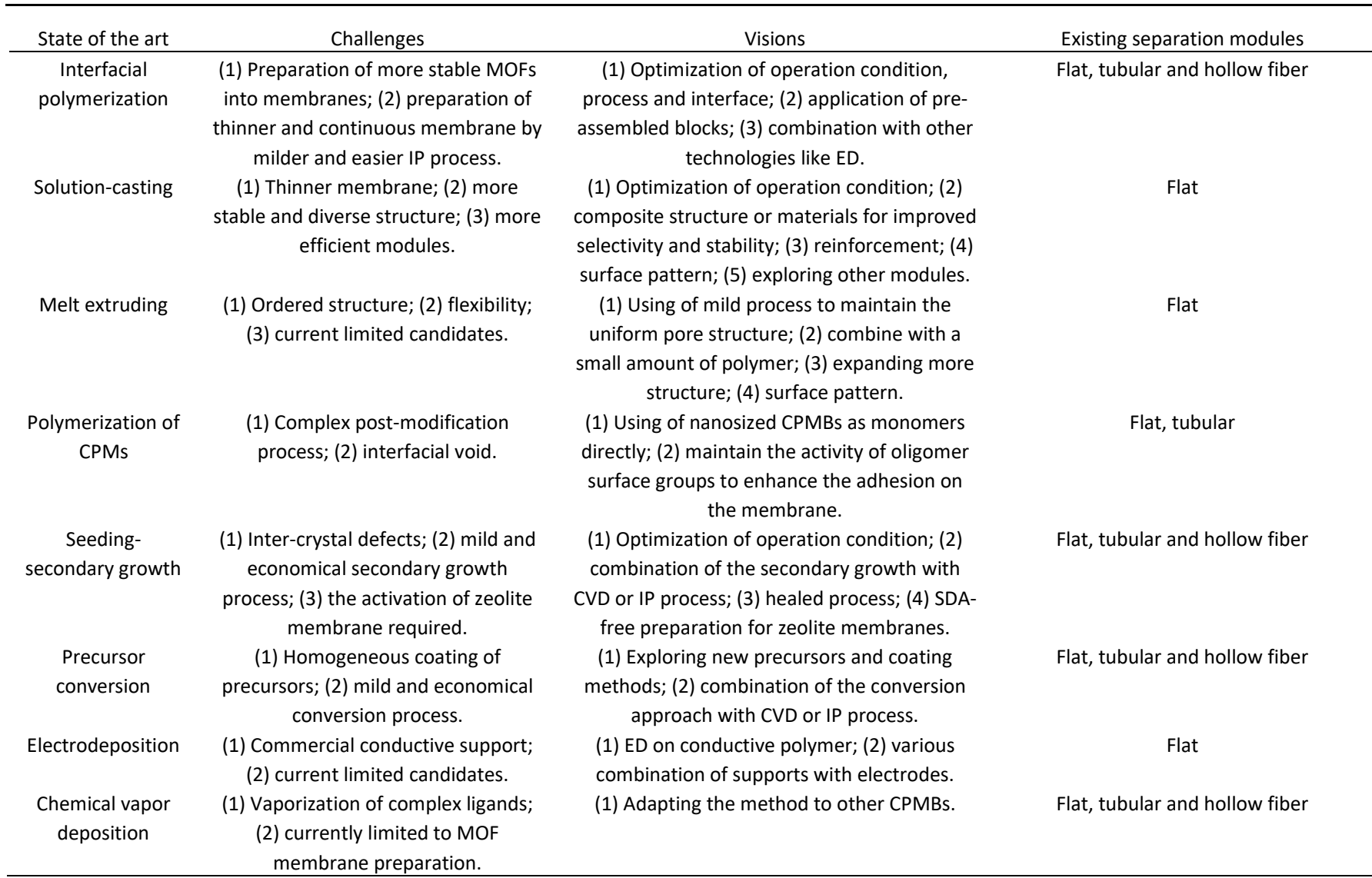

needed to fix the defects and extend the membrane lifetime of CPMBs for practical applications. ${ }^{113,223,224}$ The accumulated knowledge from the zeolite membranes has been used with modification towards preparation of MOFs membranes. While the recycling of expensive raw materials and solvents should be taken into account to reduce the cost and waste at the largescale production. The mild secondary growth condition is also alluring, in which low-cost polymer membranes can be used as supports.
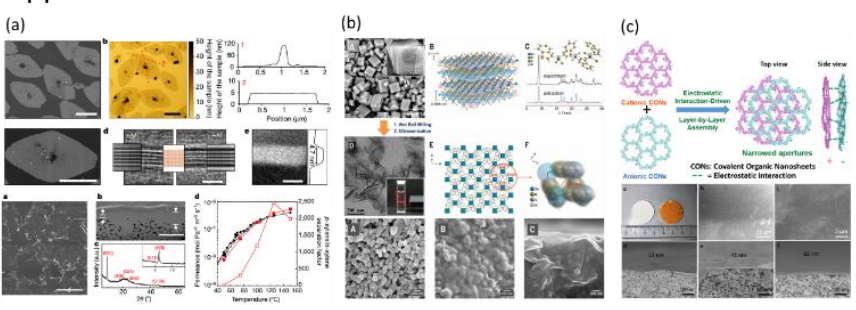

Fig. 13 Ultra-thin membrane constructed with 2D (a) zeolite, (b) MOF and (c) COF blocks. (adapted with permission from ref. ${ }^{225-227}$ )

Beyond the IP and seeding-secondary growth, the direct polymerization of CPM particles into membranes with or without the assistant of common monomers will be another appealing research filed for the scalable production of CPMBs in the future. If the common polymer monomers are involved, the nanosized functional CPMs can be applied as another monomer to interfacial polymerized into thin membranes, and the flexible membrane will be facile to process into tubular and spiral modules. The ultra-small size of CPMs should be achieved to prevent the voids between particles. When the CPM are used as the oligomers, the surface reactivity should be important to successful polymerization into continuous membranes. The capping agents can be applied to surround the CPM oligomers, then replaced by the linker of CPMs to form membrane.

On the other hand, several novel materials and technology have been developed. The new materials like POCs, HOFs and MOF glass need more systematic study (material structure, preparation process and membrane supports), and can be applied as the matrix combined with other CPMs to form allmicroporous composite membranes, due to the ease of processing. The stability and flexibility of the materials can be improved as well. For the new fabrication technology, the updating of new approaches like CVD and ED are required to expand the number of materials (CPMs) with amendable properties. Furthermore, these novel technologies can play the role of secondary growth or conversion steps in the traditional seeding-secondary growth and precursor conversion method, accelerating the membrane preparation. Recently ultrathin 
membranes with ideal performance are constructed with the blocks of 2D CPM nanosheets obtained via top-down or bottomup method (Fig. 13). ${ }^{33,} 35$ To apply these materials to the scalable membrane, the yield and purity of CPM nanosheets should be improved. The process to fabricate membranes on hollow fiber and tubular supports need to be further explored. The strategies toward healing of structural defects of membranes should be considered. We believe that with the efforts and cooperation of academia and industry, more commercial CPMBs would be available in the near future, especially to be used in the new separation fields such as chiral resolution and organic solvent nanofiltration, which are challenging for the polymer membranes.

\begin{tabular}{|c|c|}
\hline OD & Zero-dimensional \\
\hline 1D & One-dimensional \\
\hline 2D & Two-dimensional \\
\hline 3D & Three-dimensional \\
\hline 2-MIM & 2-methylimidazolate \\
\hline AAO & Anodized aluminum oxide \\
\hline ACD & Aqueously cathodic deposition \\
\hline ALD & Atomic layer deposition \\
\hline Azo & 4,4'-azodianiline \\
\hline blm & Benzimidazole \\
\hline Bpy & 2,2'-bipyridine-5,5'-diamine \\
\hline CMPs & Conjugated microporous polymers \\
\hline COFs & Covalent organic frameworks \\
\hline CPMs & Crystalline porous materials \\
\hline CPMBs & Crystalline porous membranes \\
\hline CVD & Chemical vapor deposition \\
\hline DHF & 9,9-dihexylfluorene-2,7-diamine \\
\hline ED & Electrodeposition \\
\hline EP & Electrochemical polymerization \\
\hline GO & Graphene oxide \\
\hline Н3ВТС & 1,3,5-benzenetricarboxylic acid \\
\hline HKUST & $\begin{array}{l}\text { Hong Kong university of science and } \\
\text { technology }\end{array}$ \\
\hline HOFs & Hydrogen-bonded organic frameworks \\
\hline HoP & Hot-pressing \\
\hline ILs & Ionic liquids \\
\hline IMMP & $\begin{array}{l}\text { Interfacial microfluidic membrane } \\
\text { processing }\end{array}$ \\
\hline IP & Interfacial polymerization \\
\hline LDHs & Layered double hydroxides \\
\hline MHNs & Metal hydroxide nano-strands \\
\hline MIL & Materials of Institut Lavoisier \\
\hline MMMs & Mixed matrix membranes \\
\hline MOFs & Metal-organic frameworks \\
\hline MW & Microwave \\
\hline MWCO & Molecular weight cutof \\
\hline MWRO & Molecular weight retention onset \\
\hline NF & Nanofiltration \\
\hline OSN & Organic solvent nanofiltration \\
\hline $\mathrm{Pa}$ & 1,4-phenylenediamine \\
\hline PAN & Polyacrylonitrile \\
\hline
\end{tabular}

$\begin{array}{ll}\text { PDA } & \text { Terephthalaldehyde } \\ \text { PES } & \text { Polyethersulfone } \\ \text { PI } & \text { Phase inversion } \\ \text { POCs } & \text { Porous organic cages } \\ \text { PSF } & \text { Polysulfone } \\ \text { PTSA } & \text { P-toluene sulfonic acid } \\ \text { RO } & \text { Reversed osmosis } \\ \text { SDAs } & \text { Structural directing agents } \\ \text { S.F. } & \text { Separation factor } \\ \text { SS } & \text { Stainless steel } \\ \text { TAPB } & 1,3,5 \text {-tris(4-aminophenyl)benzene } \\ \text { TFB } & 1,3,5 \text {-triformylbenzene } \\ \text { TFC } & \text { Thin-film composite } \\ \text { TFN } & \text { Thin-film nanocomposite } \\ \text { Tp } & \text { 1,3,5-triformylphloroglucinol } \\ \text { TPC } & \text { Terephthaloyl chloride } \\ \text { UF } & \text { Ultrafiltration } \\ \text { UiO } & \text { University of Oslo } \\ \text { YSZ } & \text { Yttria-stabilized zirconia } \\ \text { ZIFs } & \text { Zeolitic imidazolate frameworks }\end{array}$

\section{Conflicts of interest}

There are no conflicts to declare.

\section{Acknowledgements}

This work was supported from Thousand Talents Program for Foreign Experts (WQ20152100284), National Natural Science Foundation of China (Grants No. U1862118, 21501198, 21601205 and 21771193), Taishan Scholar Foundation (ts201511019) and the Fundamental Research Funds for the Central Universities (18CX02047A, 18CX07001A, 18CX05018A, 20CX05010A), PetroChina Innovation Foundation (2019D-50070411), Key Research and Development Projects of Shandong Province (2019JZZY010331). Sincere gratitude goes to Y. Tang Y. Wang and F. Jiao for the advice of writing, structure and artwork.

\section{References}

1. R. W. BAKER, Membrane Technology and Applications, John Wiley \& Sons, Ltd., Membrane Technology and Research, Inc. Newark, California, 2012.

2. W. J. Koros and C. Zhang, Nat Mater, 2017, 16, 289-297.

3. P. Bernardo, E. Drioli and G. Golemme, Ind. Eng. Chem. Res., 2009, 48, 4638-4663.

4. H. B. Park, J. Kamcev, L. M. Robeson, M. Elimelech and B. D. Freeman, Science, 2017, 356.

5. M. Galizia, W. S. Chi, Z. P. Smith, T. C. Merkel, R. W. Baker and B. D. Freeman, Macromolecules, 2017, 50, 7809-7843.

6. S. P. Nunes, P. Z. Culfaz-Emecen, G. Z. Ramon, T. Visser, G. H. Koops, W. Jin and M. Ulbricht, J. Membr. Sci., 2020, 598, 117761.

7. L. Wang, M. S. H. Boutilier, P. R. Kidambi, D. Jang, N. G. Hadjiconstantinou and R. Karnik, Nature nanotechnology, 2017, 12, 509-522. 
8. J. Hou, H. Zhang, G. P. Simon and H. Wang, Adv. Mater., 2019, e1902009.

9. N. Rangnekar, N. Mittal, B. Elyassi, J. Caro and M. Tsapatsis, Chem. Soc. Rev., 2015, 44, 7128-7154.

10 T. Uemura, N. Yanai and S. Kitagawa, Chem. Soc. Rev., 2009, 38, 1228-1236.

11. S. Qiu, M. Xue and G. Zhu, Chem. Soc. Rev., 2014, 43, 6116 6140.

12. H. Furukawa, K. E. Cordova, M. O'Keeffe and O. M. Yaghi, Science, 2013, 341, 1230444.

13. K. Geng, T. He, R. Liu, S. Dalapati, K. T. Tan, Z. Li, S. Tao, Y Gong, Q. Jiang and D. Jiang, Chem. Rev., 2020.

14. Z. Wang, S. Zhang, Y. Chen, Z. Zhang and S. Ma, Chem. Soc Rev., 2020, 49, 708-735.

15. M. A. Little and A. I. Cooper, Adv. Funct. Mater., 2020, 1909842

16

R. B. Lin, Y. He, P. Li, H. Wang, W. Zhou and B. Chen, Chem. Soc. Rev., 2019, 48, 1362-1389.

17. Janina Dechnik, Jorge Gascon, Christian J. Doonan, Christoph Janiak and C. J. Sumby, Angew. Chem. Int. Ed, 2017, 56, 2-21.

18. S. Yuan, X. Li, J. Zhu, G. Zhang, P. Van Puyvelde and B. Van der Bruggen, Chem. Soc. Rev., 2019, 48, 2665-2681.

19. V. M. Aceituno Melgar, J. Kim and M. R. Othman, Journal of Industrial and Engineering Chemistry, 2015, 28, 1-15.

20. J. Duan, Y. Pan, G. Liu and W. Jin, Current Opinion in Chemical Engineering, 2018, 20, 122-131.

21. J. Caro, M. Noack and P. Kolsch, Adsorption-Journal of the International Adsorption Society, 2005, 11, 215-227. X. Ma, Y. Chai, P. Li and B. Wang, Acc. Chem. Res., 2019, 52, 1461-1470.

R. Dragomirova and S. Wohlrab, Catalysts, 2015, 5, 21612222.

24. K. Adil, Y. Belmabkhout, R. S. Pillai, A. Cadiau, P. M. Bhatt, A. H. Assen, G. Maurin and M. Eddaoudi, Chem. Soc. Rev. 2017, 46, 3402-3430.

X. Li, Y. Liu, J. Wang, J. Gascon, J. Li and B. Van der Bruggen, Chem. Soc. Rev., 2017, 46, 7124-7144. X.Ma and D. Liu, Crystals, 2018, 9, 14

P. S. Goh and A. F. Ismail, Desalination, 2018, 434, 60-80. Y. He, Y. Qiao, Z. Chang and H. Zhou, Energy \& Environmental Science, 2019, 12, 2327-2344.

M. Shah, M. C. McCarthy, S. Sachdeva, A. K. Lee and H.-K. Jeong, Industrial \& Engineering Chemistry Research, 2012, 51, 2179-2199.

30. Z. Kang, L. Fan and D. Sun, J. Mater. Chem. A, 2017.

31. K. C. Khulbe, T. Matsuura, C. Y. Feng and A. F. Ismail, RSC Adv., 2016, 6, 42943-42961.

32. T. C. Bowen, R. D. Noble and J. L. Falconer, J. Membr. Sci., 2004, 245, 1-33.

33. S. Wang, L. Yang, G. He, B. Shi, Y. Li, H. Wu, R. Zhang, S. Nunes and Z. Jiang, Chem. Soc. Rev., 2020, 49, 1071-1089. C. Zhang, B. H. Wu, M. Q. Ma, Z. Wang and Z. K. Xu, Chem. Soc. Rev., 2019, 48, 3811-3841.

35. W.-g. Kim and S. Nair, Chem. Eng. Sci., 2013, 104, 908-924.

36. C. Echaide-Górriz, C. Clément, F. Cacho-Bailo, C. Téllez and J. Coronas, Journal of Materials Chemistry A, 2018, 6, 54855506.

37.

C. Wang, X. Liu, N. Keser Demir, J. P. Chen and K. Li, Chem Soc. Rev., 2016, 45, 5107-5134.

38. X. Liu, X. Wang and F. Kapteijn, Chem. Rev., 2020.
39.

40.

41.

43.

44

45.

46.

47.

48.

48.

49.

50.

51.

51.

52.

53.

54.

54.

56.

58.

59.

60.

\section{1.}

62.

63.

64.

65.

66.

67.
Y. Morigami, M. Kondo, J. Abe, H. Kita and K. Okamoto, Sep. Purif. Technol., 2001, 25, 251-260.

J. Gascon, F. Kapteijn, B. Zornoza, V. Sebastián, C. Casado and J. Coronas, Chem. Mater., 2012, 24, 2829-2844.

J. Yu and R. Xu, Acc. Chem. Res., 2003, 36, 481-490.

V. Valtchev, G. Majano, S. Mintova and J. Perez-Ramirez, Chem. Soc. Rev., 2013, 42, 263-290.

H. Y. Jiang, B. Q. Zhang, Y. S. Lin and Y. D. Li, Chin. Sci. Bull., 2004, 49, 2547-2554.

S. Yang, Y. Chiang and S. Nair, Energy Technology, 2019, 7, 1900494.

Y. S. Lin and M. C. Duke, Current Opinion in Chemical Engineering, 2013, 2, 209-216.

S. Kitagawa, R. Kitaura and S. Noro, Angewandte ChemieInternational Edition, 2004, 43, 2334-2375.

Z. Wang, Q. Ge, J. Shao and Y. Yan, J. Am. Chem. Soc., 2009, 131, 6910-6911.

Q. Ge, Z. Wang and Y. Yan, J. Am. Chem. Soc., 2009, 131 17056-17057.

X. Gao, B. Gao, H. Liu, C. Zhang, Y. Zhang, J. Jiang and X. Gu, Sep. Purif. Technol., 2020, 234, 116121.

X. Wang, Y. Chen, C. Zhang, X. Gu and N. Xu, J. Membr. Sci., 2014, 455, 294-304.

Y. S. Li, H. L. Chen, J. Liu and W. S. Yang, J. Membr. Sci., 2006, 277, 230-239.

Z. P. Lai, G. Bonilla, I. Diaz, J. G. Nery, K. Sujaoti, M. A. Amat, E. Kokkoli, O. Terasaki, R. W. Thompson, M. Tsapatsis and D. G. Vlachos, Science, 2003, 300, 456-460.

P. Tung Cao Thanh, H. S. Kim and K. B. Yoon, Science, 2011, 334, 1533-1538.

T. C. Pham, T. H. Nguyen and K. B. Yoon, Angew. Chem. Int. Ed. Engl., 2013, 52, 8693-8698.

K. V. Agrawal, B. Topuz, T. C. Pham, T. H. Nguyen, N. Sauer, N. Rangnekar, H. Zhang, K. Narasimharao, S. N. Basahel, L. F. Francis, C. W. Macosko, S. Al-Thabaiti, M. Tsapatsis and K. B. Yoon, Adv. Mater., 2015, 27, 3243-3249.

M. Matsukata, N. Nishiyama and K. Ueyama, J. Chem. Soc., Chem. Commun., 1994, 339-340.

S. Alfaro, M. Arruebo, J. I. Coronas, M. Menendez and J. S. Ia, Microporous Mesoporous Mater., 2001, 50, 195 - 200. H. Guo, G. Kong, G. Yang, J. Pang, Z. Kang, S. Feng, L. Zhao, L. Fan, L. Zhu, A. Vicente, P. Peng, Z. Yan, D. Sun and S. Mintova, Angew. Chem. Int. Ed. Engl., 2020, 59, 6284-6288. A. Huang and W. Yang, Microporous Mesoporous Mater., 2007, 102, 58-69.

C. Gu, N. Hosono, J.-J. Zheng, Y. Sato, S. Kusaka, S. Sakaki and S. Kitagawa, Science 2019, 363, 387-391.

G. Férey and C. Serre, Chem. Soc. Rev., 2009, 38, 1380-1399 Z. Chang, D. H. Yang, J. Xu, T. L. Hu and X. H. Bu, Adv. Mater., 2015, 27, 5432-5441.

Y. Wang, H. Jin, Q. Ma, K. Mo, H. Mao, A. Feldhoff, X. Cao, Y. Li, F. Pan and Z. Jiang, Angew. Chem. Int. Ed, 2020, 132, 4395-4399.

Z. Kang, M. Xue, L. Fan, J. Ding, L. Guo, L. Gao and S. Qiu, Chem. Commun., 2013, 49, 10569-10571.

M. Kalaj, K. C. Bentz, S. Ayala, Jr., J. M. Palomba, K. S. Barcus, Y. Katayama and S. M. Cohen, Chem. Rev., 2020.

X. Liu, N. K. Demir, Z. Wu and K. Li, J. Am. Chem. Soc., 2015, 137, 6999-7002.

W. Zhang, Y. Hu, J. Ge, H. L. Jiang and S. H. Yu, J. Am. Chem. Soc., 2014, 136, 16978-16981. 
68

J. M. Lee and A. I. Cooper, Chem. Rev., 2020, 120, 21712214.

69. J. Yao, D. Dong, D. Li, L. He, G. Xu and H. Wang, Chem. Commun., 2011, 47, 2559-2561.

70. H. T. Kwon and H.-K. Jeong, J. Am. Chem. Soc., 2013, 135, 10763-10768.

71. A. J. Brown, N. A. Brunelli, K. Eum, F. Rashidi, J. R. Johnson, W. J. Koros, C. W. Jones and S. Nair, Science, 2014, 345, 72 75.

72

Y. Li, L. H. Wee, A. Volodin, J. A. Martens and I. F. Vankelecom, Chem Commun (Camb), 2015, 51, 918-920. Y. Li, L. H. Wee, J. A. Martens and I. F. J. Vankelecom, J. Membr. Sci., 2017, 523, 561-566.

74

J. Campbell, R. P. Davies, D. C. Braddock and A. G. Livingston, Journal of Materials Chemistry A, 2015, 3, 96689674.

75

X. Zhang, Y. Li, C. Van Goethem, K. Wan, W. Zhang, J. Luo, I. F. J. Vankelecom and J. Fransaer, Matter, 2019, 1, 1285 1292.

76.

Y. Zhang, X. Feng, H. Li, Y. Chen, J. Zhao, S. Wang, L. Wang and B. Wang, Angew. Chem. Int. Ed., 2015, 54, 4259-4263. W. Wu, M. Jia, J. Su, Z. Li and W. Li, AlChE J., 2020.

78 Y. Sun, Y. Liu, J. Caro, X. Guo, C. Song and Y. Liu, Angew. Chem. Int. Ed., 2018, 57, 16088-16093.

79. Y. Mao, L. shi, H. Huang, W. Cao, J. Li, L. Sun, X. Jin and X Peng, Chem. Commun., 2013, 49, 5666-5668.

80. Y. Mao, J. Li, W. Cao, Y. Ying, L. Sun and X. Peng, ACS applied materials \& interfaces, 2014, 6, 4473-4479.

81

J. Yu, Y. Pan, C. Wang and Z. Lai, Chem. Eng. Sci., 2016, 141 119-124.

82. W. Li, P. Su, Z. Li, Z. Xu, F. Wang, H. Ou, J. Zhang, G. Zhang and E. Zeng, Nat Commun, 2017, 8, 406.

83. Y. Liu, J. H. Pan, N. Wang, F. Steinbach, X. Liu and J. Caro, Angew. Chem. Int. Ed. Engl., 2015, 54, 3028-3032.

84 Y. Liu, N. Wang, J. H. Pan, F. Steinbach and J. Caro, J. Am. Chem. Soc., 2014, 136, 14353-14356.

85. Y. Li, H. Liu, H. Wang, J. Qiu and X. Zhang, Chemical science, 2018, 9, 4132-4141.

86. P. Nian, H. Liu and X. Zhang, J. Membr. Sci., 2019, 573, 200209.

87.

W. Li, J. Shi, Z. Li, W. Wu, Y. Xia, Y. Yu and G. Zhang, Advanced Materials Interfaces, 2018, 5, 1800032

88. Z. Kang, S. Wang, R. Wang, H. Guo, B. Xu, S. Feng, L. Fan, L. Zhu, W. Kang, J. Pang, H. Sun, X. Du, M. Zhang and D. Sun, Materials Chemistry Frontiers, 2018, 2, 1911-1919.

89. G. He, M. Dakhchoune, J. Zhao, S. Huang and K. V. Agrawal, Adv. Funct. Mater., 2018, 28, 1707427.

90. S. Zhou, Y. Wei, L. Li, Y. Duan, Q. Hou, L. Zhang, L.-X. Ding J. Xue, H. Wang and J. Caro, Science advances, 2018, 4, eaau1393.

91. Q. Hou, Y. Wu, S. Zhou, Y. Wei, J. Caro and H. Wang, Angew. Chem. Int. Ed, 2018, 58, 327-331.

92. Q. Hou, S. Zhou, Y. Wei, J. Caro and H. Wang, J. Am. Chem. Soc., 2020.

93. R. Wei, H. Y. Chi, X. Li, D. Lu, Y. Wan, C. W. Yang and Z. Lai, Adv. Funct. Mater., 2019, 30, 1907089.

94. X. Ma, P. Kumar, N. Mittal, A. Khlyustova, P. Daoutidis, K. A. Mkhoyan and M. Tsapatsis, Science, 2018, 361, 10081011.

95. K. Dey, M. Pal, K. C. Rout, H. S. Kunjattu, A. Das, R. Mukherjee, U. K. Kharul and R. Banerjee, J. Am. Chem. Soc., 2017, 139, 13083-13091.
96.

M. Matsumoto, L. Valentino, G. M. Stiehl, H. B. Balch, A. R. Corcos, F. Wang, D. C. Ralph, B. J. Mariñas and W. R. Dichtel, Chem, 2018, 4, 308-317.

97. L. Valentino, M. Matsumoto, W. R. Dichtel and B. J. Marinas, Environ. Sci. Technol., 2017, 51, 14352-14359.

$98 . \quad$ D. B. Shinde, G. Sheng, X. Li, M. Ostwal, A. H. Emwas, K. W. Huang and Z. Lai, J. Am. Chem. Soc., 2018, 140, 1434214349.

99. N. A. Khan, R. Zhang, H. Wu, J. Shen, J. Yuan, C. Fan, L. Cao, M. A. Olson and Z. Jiang, J. Am. Chem. Soc., 2020.

100. R. Wang, X. Shi, A. Xiao, W. Zhou and Y. Wang, J. Membr. Sci., 2018, 566, 197-204.

101. Q. T. Le, D. H. Nguyen, N. M. Nguyen, D. P. Nguyen, T. M. Nguyen, T. N. Nguyen and T. C. Pham, ChemSusChem, 2020, 13, 1720-1724.

102. H. S. Sasmal, A. Halder, H. S. Kunjattu, K. Dey, A. Nadol, T. G. Ajithkumar, P. Ravindra Bedadur and R. Banerjee, J. Am. Chem. Soc., 2019, 141, 20371-20379.

103. Q. Song, S. Jiang, T. Hasell, M. Liu, S. Sun, A. K. Cheetham, E. Sivaniah and A. I. Cooper, Adv. Mater., 2016, 28, 26292637.

104. Y. Zhang, X. Zhang, Z. Chen, K. I. Otake, G. W. Peterson, Y. Chen, X. Wang, L. R. Redfern, S. Goswami, P. Li, T. Islamoglu, B. Wang and O. K. Farha, ChemSusChem, 2020, 13, 1710-1714.

105. Z. Zhai, C. Jiang, N. Zhao, W. Dong, P. Li, H. Sun and Q. J. Niu, J. Membr. Sci., 2020, 595, 117505.

106. T. Ben, H. Ren, S. Ma, D. Cao, J. Lan, X. Jing, W. Wang, J. Xu, F. Deng, J. M. Simmons, S. Qiu and G. Zhu, Angew. Chem. Int. Ed. Engl., 2009, 48, 9457-9460.

107. Y. Tian and G. Zhu, Chem. Rev., 2020.

108. L. Chen, P. S. Reiss, S. Y. Chong, D. Holden, K. E. Jelfs, T. Hasell, M. A. Little, A. Kewley, M. E. Briggs, A. Stephenson, K. M. Thomas, J. A. Armstrong, J. Bell, J. Busto, R. Noel, J. Liu, D. M. Strachan, P. K. Thallapally and A. I. Cooper, Nat Mater, 2014, 13, 954-960.

109. T. Hasell and A. I. Cooper, Nature Reviews Materials, 2016 , 1, 16053.

Y. He, S. Xiang and B. Chen, J. Am. Chem. Soc., 2011, 133, 14570-14573.

111. F. Hu, C. Liu, M. Wu, J. Pang, F. Jiang, D. Yuan and M. Hong, Angew. Chem. Int. Ed. Engl., 2017, 56, 2101-2104.

112. S. Loeb and S. Sourirajan, Sea water demineralization by means of an osmotic membrane, American Chemical Society, Washington, DC, 1963.

113. S. Feng, Y. Shang, Z. Wang, Z. Kang, R. Wang, J. Jiang, L. Fan, W. Fan, Z. Liu, G. Kong, Y. Feng, S. Hu, H. Guo and D. Sun, Angew. Chem. Int. Ed., 2020, 59, 3840-3845.

114. F. Zhang, J.-b. Fan and S. Wang, Angew. Chem. Int. Ed, 2020, 59.

R. Ameloot, F. Vermoortele, W. Vanhove, M. B. Roeffaers, B. F. Sels and D. E. De Vos, Nat Chem, 2011, 3, 382-387. S. Karan, Zhiwei Jiang and A. G. Livingston, Science, 2015, 348, 1347-1351.

117. P. M. Budd, E. S. Elabas, B. S. Ghanem, S. Makhseed, N. B. McKeown, K. J. Msayib, C. E. Tattershall and a. D. Wang, Adv. Mater., 2004, 16, 456-459.

118. A. Knebel, A. Bavykina, S. J. Datta, L. Sundermann, L. Garzon-Tovar, Y. Lebedev, S. Durini, R. Ahmad, S. M. Kozlov, G. Shterk, M. Karunakaran, I. D. Carja, D. Simic, I. Weilert, M. Kluppel, U. Giese, L. Cavallo, M. Rueping, M. Eddaoudi, J. Caro and J. Gascon, Nat Mater, 2020. 
119. B. D. Egleston, K. V. Luzyanin, M. C. Brand, R. Clowes, M. E. Briggs, R. L. Greenaway and A. I. Cooper, Angew Chem Int Ed Engl., 2020, 59.

120. Y. S. Li, H. Bux, A. Feldhoff, G. L. Li, W. S. Yang and J. Caro, Adv. Mater., 2010, 22, 3322-3326.

121. F. Zhang, X. Zou, X. Gao, S. Fan, F. Sun, H. Ren and G. Zhu, Adv. Funct. Mater., 2012, 22, 3583-3590.

122. A. Huang, H. Bux, F. Steinbach and J. Caro, Angew. Chem. Int. Ed., 2010, 49, 4958-4961.

123. A. Huang and J. Caro, Angew. Chem. Int. Ed., 2011, 50, 4979-4982.

124. X. J. Yin, G. S. Zhu, W. S. Yang, Y. S. Li, G. Q. Zhu, R. Xu, J. Y. Sun, S. L. Qiu and R. R. Xu, Adv. Mater., 2005, 17, 2006-+.

125. J. T. Jones, T. Hasell, X. Wu, J. Bacsa, K. E. Jelfs, M. Schmidtmann, S. Y. Chong, D. J. Adams, A. Trewin, F. Schiffman, F. Cora, B. Slater, A. Steiner, G. M. Day and A. I. Cooper, Nature, 2011, 474, 367-371.

126. T. Hasell, S. Y. Chong, M. Schmidtmann, D. J. Adams and A. I. Cooper, Angew. Chem. Int. Ed. Engl., 2012, 51, 71547157.

127. K. Ma, P. Li, J. H. Xin, Y. Chen, Z. Chen, S. Goswami, X. Liu, S. Kato, H. Chen, X. Zhang, J. Bai, M. C. Wasson, R. R. Maldonado, R. Q. Snurr and O. K. Farha, Cell Reports Physical Science, 2020, 1, 100024.

128. A. L. McGaughey, P. Karandikar, M. Gupta and A. E. Childress, ACS Applied Polymer Materials, 2020, 2, 12561267.

129. K. Xie, Q. Fu, C. Xu, H. Lu, Q. Zhao, R. Curtain, D. Gu, P. A. Webley and G. G. Qiao, Energy \& Environmental Science, 2018, 11, 544-550.

130. Z. Kang, S. Wang, L. Fan, Z. Xiao, R. Wang and D. Sun, Mater Lett., 2017, 189, 82-85.

131. W. Liu, S. D. Jiang, Y. Yan, W. Wang, J. Li, K. Leng, S. Japip J. Liu, H. Xu, Y. Liu, I. H. Park, Y. Bao, W. Yu, M. D. Guiver, S Zhang and K. P. Loh, Nat Commun, 2020, 11, 1633.

132. S. Horike, S. S. Nagarkar, T. Ogawa and S. Kitagawa, Angew. Chem. Int. Ed. Engl., 2020, 59, 6652-6664.

133. K. J. Mackenzie, Film and sheeting material, Kirk-Othmer Encyclopedia of Chemical Technology, John Wiley \& Sons, Inc., Hoboken, NJ, , 1992.

134. Y. Zhao, S. Y. Lee, N. Becknell, O. M. Yaghi and C. A. Angell, J. Am. Chem. Soc., 2016, 138, 10818-10821.

135. T. D. Bennett and S. Horike, Nature Reviews Materials, 2018, 3, 431-440.

136. J. Hou, A. F. Sapnik and T. D. Bennett, Chemical science, 2020, 11, 310-323

137. A. M. Bumstead, M. F. Thorne and T. D. Bennett, Faraday Discuss., 2020.

138. A. Qiao, T. D. Bennett, H. Tao, A. Krajnc, G. Mali, C. M. Doherty, A. W. Thornton, J. C. Mauro, G. N. Greaves and Y. Yue, Science advances, 2018, 4, eaao6827.

139. S. Li, R. Limbach, L. Longley, A. A. Shirzadi, J. C. Walmsley, D. N. Johnstone, P. A. Midgley, L. Wondraczek and T. D. Bennett, J. Am. Chem. Soc., 2019, 141, 1027-1034.

140. Yaqi Wu, Shu Zeng, Danhua Yuan, Jiacheng Xing, Hanbang Liu, Shutao Xu, Yingxu Wei, Yunpeng Xu and Z. Liu, Angew. Chem. Int. Ed., 2020, 59, $6765-6768$.

141. Z. Guo, W. Zheng, X. Yan, Y. Dai, X. Ruan, X. Yang, X. Li, N. Zhang and G. He, J. Membr. Sci., 2020, 605, 118101.

142. T. Kitao, Y. Zhang, S. Kitagawa, B. Wang and T. Uemura, Chem. Soc. Rev., 2017, 46, 3108-3133.
143.

144.

Zhou, X. Feng, X. Ma and B. Wang, Adv. Mater., 2017.

Y. Chen, S. Li, X. Pei, J. Zhou, X. Feng, S. Zhang, Y. Cheng, H. Li, R. Han and B. Wang, Angew.Chem. Int.Ed., 2015, 55, $3419-3423$.

145. K. Dey, S. K. H, A. M. Chahande and R. Banerjee, Angew. Chem. Int. Ed., 2020, 59, $1161-1165$

146.

Y. Katayama, M. Kalaj, K. S. Barcus and S. M. Cohen, J. Am. Chem. Soc., 2019, 141, 20000-20003.

147.

M. Kalaj, M. S. Denny, Jr., K. C. Bentz, J. M. Palomba and S. M. Cohen, Angew. Chem. Int. Ed. Engl., 2019, 58, 23362340.

148. Z. Yin, C. Cui, H. Chen, Duoni, X. Yu and W. Qian, Small, 2020, 16, e1902301.

149. S. Chatterjee, N. Y. Kim, N. M. Pugno, M. Biswal, B. V. Cunning, M. Goo, S. Jin, S. H. Lee, Z. Lee and R. S. Ruoff, Chem. Mater., 2020, 32, 3134-3143.

150. Y.-H. Yao, J. Li, H. Zhang, H.-L. Tang, L. Fang, G.-D. Niu, X. J. Sun and F.-M. Zhang, Journal of Materials Chemistry $A$, 2020.

151. X. Gao, J. Zhang, K. Huang and J. Zhang, ACS Appl. Mater. Interfaces, 2018, 10, 34640-34645.

152. P. Duan, J. C. Moreton, S. R. Tavares, R. Semino, G. Maurin S. M. Cohen and K. Schmidt-Rohr, J. Am. Chem. Soc., 2019, 141, 7589-7595.

153. Z. Liu, C. Shao, B. Jin, Z. Zhang, Y. Zhao, X. Xu and R. Tang, Nature, 2019, 574, 394-398.

154. H. Guo, Y. Zhu, S. Qiu, J. A. Lercher and H. Zhang, Adv. Mater., 2010, 22, 4190-4192.

155. T. Tsuruoka, S. Furukawa, Y. Takashima, K. Yoshida, S. Isoda and S. Kitagawa, Angew. Chem. Int. Ed., 2009, 48, 47394743.

156. Yuko Takata, T. Tsuru, T. Yoshioka and M. Asaeda, Microporous Mesoporous Mater., 2002, 54 257-268.

157. Y. Morigami, M. Kondo, J. Abe, H. Kita and K. Okamoto, Sep. Purif. Technol., 2001, 25, 251-260.

158. P. R. H. P. Rao and M. Matsukata, Chem. Commun., 1996, 1441-1442.

159. M. Zhang, X. Jing, S. Zhao, P. Shao, Y. Zhang, S. Yuan, Y. Li, C. Gu, X. Wang, Y. Ye, X. Feng and B. Wang, Angew. Chem. Int. $E d, 2019, \mathbf{5 8}, 8768-8772$.

160. S. Mintova, M. Jaber and V. Valtchev, Chem. Soc. Rev., 2015, 44, 7207-7233.

161. H. Guo, G. Zhu, H. Li, X. Zou, X. Yin, W. Yang, S. Qiu and R. $\mathrm{Xu}$, Angewandte Chemie-International Edition, 2006, 45, 7053-7056.

162.

Tatiana Gallego-Lizona, Emma Edwardsa, Giuseppe Lobiundob and L. F. d. Santos, J. Membr. Sci., 2002, 197, 309-319.

163. M. Ji, X. Gao, X. Wang, Y. Zhang, J. Jiang and X. Gu, J. Membr. Sci., 2018, 563, 460-469.

164. K. B. Yoon, Acc. Chem. Res., 2007, 40, 29-40

165. Y. Peng, Z. Zhan, L. Shan, X. Li, Z. Wang and Y. Yan, J. Membr. Sci., 2013, 444, 60-69.

166. K. Kusakabe, T. Kuroda, A. Murata and S. Morooka, Ind Eng. Chem. Res., 1997, 36, 649-655.

167. S. Mintova and T. Bein, Adv. Mater., 2001, 13, 1880-1883.

168. M. P. Bernal, G. Xomeritakis and M. Tsapatsis, Catal. Today, 2001, 67, 101-107.

A. S. Huang, Y. S. Lin and W. S. Yang, J. Membr. Sci., 2004, 245, 41-51. 
170. Z. Wang, J. Shao and Q. Ge, Petrochemical Technology, 2010, 39, 583-591.

171. X. Wang, Z. Yang, C. Yu, L. Yin, C. Zhang and X. Gu, Microporous Mesoporous Mater., 2014, 197, 17-25.

172. M. Kanezashi, J. O' Brien and Y.S. Lin, J. Membr. Sci., 2006, 286, 213-222.

173. J. White, P. K. Dutta, K. Shqau and H. Verweij, Microporous Mesoporous Mater., 2008, 115, 389-398.

174. H. Richter, I. Voigt and J. T. Kühnert, Desalination 2006, 199, 92-93.

175. C. Yu, Y. Liu, G. Chen, X. Gu and W. Xing, Chin. J. Chem. Eng 2011, 19, 904-910.

176. T. Gallego-Lizon, E. Edwards, G. Lobiundo and L. F. d. Santos, J. Membr. Sci., 2002, 197, 309-319.

177. L. Shan, J. Shao, Z. Wang and Y. Yan, J. Membr. Sci., 2011, 378, 319-329.

178. Q. Ge, Z. Wang and Y. Yan, J. Am. Chem. Soc., 2009, 131, 17056-17057.

179. K. P. Dey, D. Kundu, M. Chatterjee and M. K. Naskar, J. Am. Ceram. Soc., 2013, 96, 68-72.

180. A. Huang, N. Wang and J. Caro, Micropor. Mesopor. Mater. , 2012, 164, 294-301.

181. C. Yuan, Q. Liu, H. Chen and A. Huang, RSC Adv., 2014, 4, 41982-41988.

182. A. Huang and J. Caro, J. Mater. Chem., 2011, 21, 1142411429.

183. S. Shirazian and S. N. Ashrafizadeh, Fuel, 2015, 148, 112119.

184. L. Lang, X. Liu and B. Zhang, Appl. Surf. Sci., 2009, 255, 4886-4890.

185. M. K. Naskar, D. Kundu and M. Chatterjee, J. Porous Mater., 2011, 18, 319-327.

186. Y. Li and W. Yang, J. Membr. Sci., 2008, 316, 3-17.

187. Y. Li, J. Liu, H. Chen, W. Yang and L. Lin, Chinese Journal of Catalysis, 2006, 27, 544-546.

188. Li SG, Carreon MA, Zhang YF, Funek HH, Noble RD and F. JL:, J. Membr Sci 2010, 352, 7-13.

189. J. Wang, P. Ye, X. Gao, Y. Zhang and X. Gu, Chin. J. Chem. Eng., 2018, 26, 1477-1484.

190. Z. Kang, M. Xue, L. Fan, L. Huang, L. Guo, G. Wei, B. Chen and S. Qiu, Energy Environ. Sci., 2014, 7, 4053-4060.

191. Y. Mao, J. Li, W. Cao, Y. Ying, P. Hu, Y. Liu, L. Sun, H. Wang, C. Jin and X. Peng, Nat. Commun., 2014, 5, 5532.

192. H. Awala, J. P. Gilson, R. Retoux, P. Boullay, J. M. Goupil, V. Valtchev and S. Mintova, Nat Mater, 2015, 14, 447-451.

193. N. Eng-Poh, D. Chateigner, T. Bein, V. Valtchev and S. Mintova, Science, 2012, 335, 70-73.

194. G. Yang, H. Guo, Z. Kang, L. Zhao, S. Feng, F. Jiao and S. Mintova, ChemSusChem, 2018.

195. Y. Hu, X. Dong, J. Nan, W. Jin, X. Ren, N. Xu and Y. M. Lee, Chem. Commun., 2011, 47, 737-739.

196. J. Nan, X. Dong, W. Wang, W. Jin and N. Xu, Langmuir : the ACS journal of surfaces and colloids, 2011, 27, 4309-4312.

197. Y. Jian-feng, Z. Li-xiong and X. Nan-ping, JOURNAL OF NANIING UNIVERSITY OF TECHNOLOGY, 2004, 1, 103-109.

198. W. Xu, J. Dong, J. Li, J. Li and F. Wu, J. Chem. Soc., Chem. Commun., 1990, 755-756

199. J. Dong, Tao Dou, Xinsheng Zhao and L. Gao, J. Chem. Soc. Chem. Commun., 1992, 1056-1058.

200. Norikazu Nishiyama, Korekazu Ueyama and M. Matsukata, Microporous Mater., 1996, 7 299-308.
201.

202.

203.

204.

205.

206.

207.

208. 209.

210.

211.

213.

214.

216.

218.

219.

220.

221.

222.

223.

224.

225.
Y. He, X.-m. Cui, X.-d. Liu, Y.-p. Wang, J. Zhang and K. Liu, J. Membr. Sci., 2013, 447, 66-72.

K. Ueno, H. Negishi, T. Okuno, H. Tawarayama, S. Ishikawa, M. Miyamoto, S. Uemiya and Y. Oumi, Microporous Mesoporous Mater., 2019, 289, 109645.

. Guo, G. Zhu, I. J. Hewitt and S. Qiu, J. Am. Chem. Soc. 2009, 131, 1646-+.

X. Zou, G. Zhu, I. J. Hewitt, F. Sun and S. Qiu, Dalton transactions, 2009, 3009-3013.

Y. Mao, B. Su, W. Cao, J. Li, Y. Ying, W. Ying, Y. Hou, L. Sun and X. Peng, ACS applied materials \& interfaces, 2014, 6, 15676-15685.

J. Li, W. Cao, Y. Mao, Y. Ying, L. Sun and X. Peng, CrystEngComm, 2014, 16, 9788-9791.

W. Li, Q. Meng, X. Li, C. Zhang, Z. Fan and G. Zhang, Chem Commun (Camb), 2014, 50, 9711-9713.

Y. Liu, Y. Ban and W. Yang, Adv. Mater., 2017, 29.

W. Li, Y. Zhang, P. Su, Z. Xu, G. Zhang, C. Shen and Q. Meng, J. Mater. Chem. A, 2016, 4, 18747-18752.

Z. Kang, S. Wang, L. Fan, M. Zhang, W. Kang, J. Pang, X. Du, H. Guo, R. Wang and D. Sun, Communications Chemistry, 2018, 1.

C. Gu, Y. Chen, Z. Zhang, S. Xue, S. Sun, K. Zhang, C. Zhong, H. Zhang, Y. Pan, Y. Lv, Y. Yang, F. Li, S. Zhang, F. Huang and Y. Ma, Adv. Mater., 2013, 25, 3443-3448.

T. Yu, W. Chu, R. Cai, Y. Liu and W. Yang, Angew. Chem. Int. Ed. Engl., 2015, 54, 13032-13035.

G. H. A. Therese and P. V. Kamath, Chem. Mater., 2000, 12, 1195-120412.

Á. Berenguer-Murcia, E. Morallón, D. Cazorla-Amorós and Á. Linares-Solano, Microporous Mesoporous Mater., 2003, 66, 331-340.

A. Knebel, B. Geppert, K. Volgmann, D. I. Kolokolov, A. G. Stepanov, J. Twiefel, P. Heitjans, D. Volkmer and J. Caro, Science, 2017, 358, 347-351.

I. Stassen, M. Styles, G. Grenci, H. V. Gorp, W. Vanderlinden, S. D. Feyter, P. Falcaro, D. D. Vos, P. Vereecken and R. Ameloot, Nat Mater, 2016, 15, 304-310. W.-J. Li, M. Tu, R. Cao and R. A. Fischer, J. Mater. Chem. A, 2016, 4, 12356-12369.

W. Li, Y. Zhang, C. Zhang, Q. Meng, Z. Xu, P. Su, Q. Li, C. Shen, Z. Fan, L. Qin and G. Zhang, Nat. Commun., 2016, 7, 11315.

W. Wu, J. Su, M. Jia, Z. Li, G. Liu and W. Li, Sci. Adv. , 2020, 6, eaax7270.

M. Kandiah, M. H. Nilsen, S. Usseglio, S. Jakobsen, U. Olsbye, M. Tilset, C. Larabi, E. A. Quadrelli, F. Bonino and K. P. Lillerud, Chem. Mater., 2010, 22, 6632-6640.

T. Loiseau, C. Serre, C. Huguenard, G. Fink, F. Taulelle, M. Henry, T. Bataille and G. Ferey, Chem.-Eur. J., 2004, 10, 1373-1382.

J. Hao, D. J. Babu, Q. Liu, H.-Y. Chi, C. Lu, Y. Liu and K. V. Agrawal, Journal of Materials Chemistry A, 2020, 8, 76337640.

X. Wang, L. Zhai, Y. Wang, R. Li, X. Gu, Y. D. Yuan, Y. Qian, Z. Hu and D. Zhao, ACS applied materials \& interfaces, 2017, 9, 37848-37855.

S. Hong, D. Kim, Y. Jeong, E. Kim, J. C. Jung, N. Choi, J. Nam, A. C. K. Yip and J. Choi, Chem. Mater., 2018, 30, 3346-3358. M. Y. Jeon, D. Kim, P. Kumar, P. S. Lee, N. Rangnekar, P. Bai, M. Shete, B. Elyassi, H. S. Lee, K. Narasimharao, S. N. Basahel, S. Al-Thabaiti, W. Xu, H. J. Cho, E. O. Fetisov, R. 
Journal Name

Thyagarajan, R. F. DeJaco, W. Fan, K. A. Mkhoyan, J. I. Siepmann and M. Tsapatsis, Nature, 2017, 543, 690-694.

226. Yuan Peng, Y. Li, Y. Ban, H. Jin, W. Jiao, X. Liu and W. Yang, Science, 2014, 346, 1356-1359.

227. Y. Ying, M. Tong, S. Ning, S. K. Ravi, S. B. Peh, S. C. Tan, S. J. Pennycook and D. Zhao, J. Am. Chem. Soc., 2020, 142, 4472-4480. 